Supplementary Information

\title{
Just add water: modulating the structure-derived acidity of catalytic hexameric resorcinarene capsules
}

David A. Poole III, Simon Mathew, Joost N. H. Reek*

Homogeneous, Supramolecular, and Bioinspired Catalysis group, van't Hoff Institute for Molecular Science (HIMS), University of Amsterdam (UvA), Science Park 904, 1098 XH Amsterdam, The Netherlands 


\section{Contents:}

1. Forcefield parameterization of resorcin[4] arene structures .................................................................

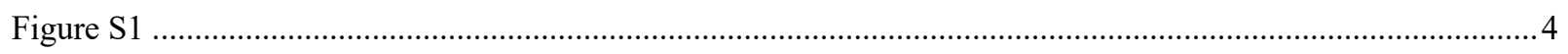

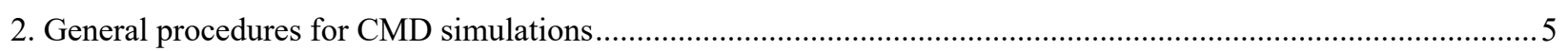

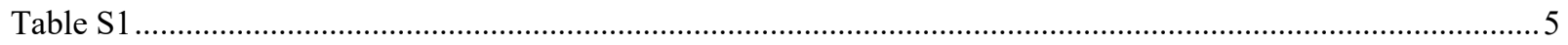

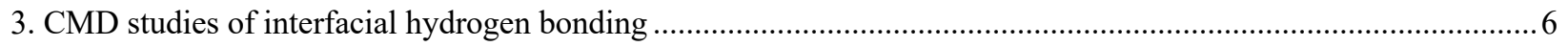

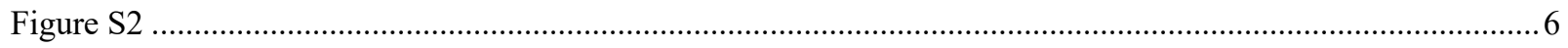

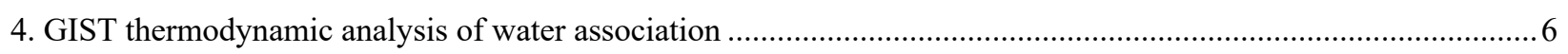

5. Materials

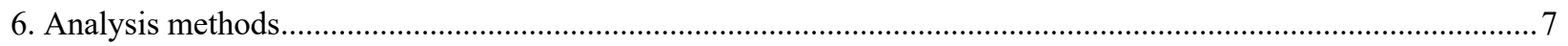

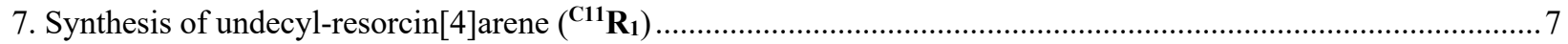

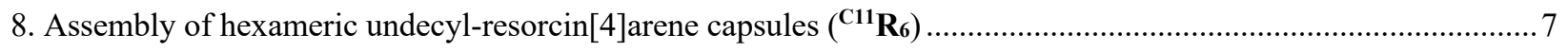

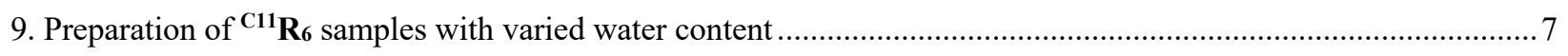

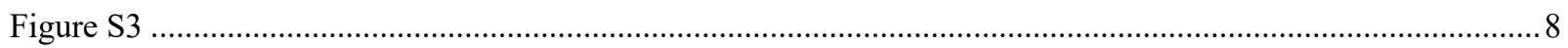

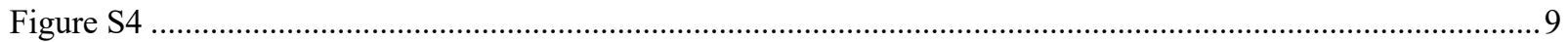

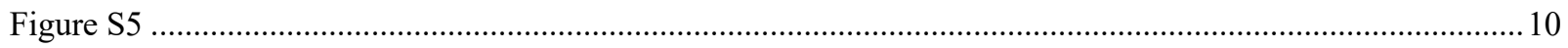

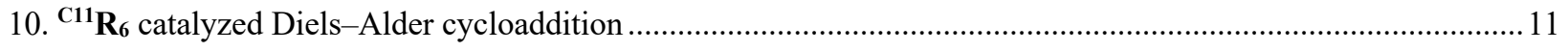

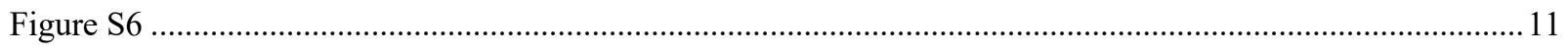

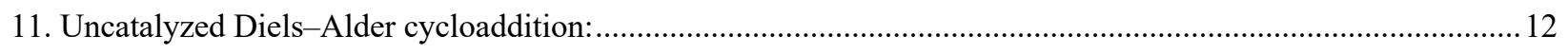

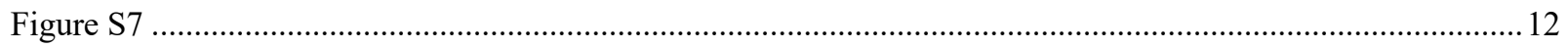

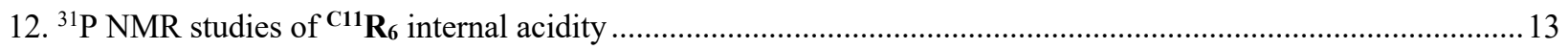

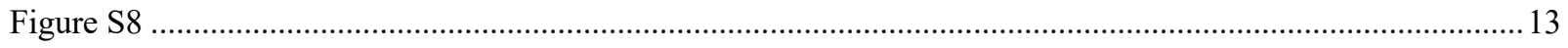

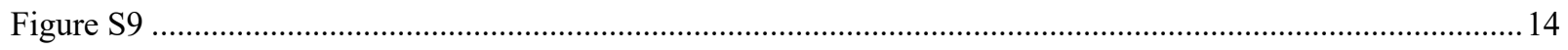

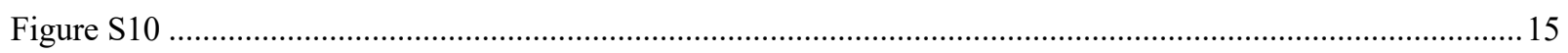

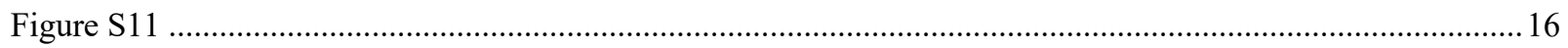

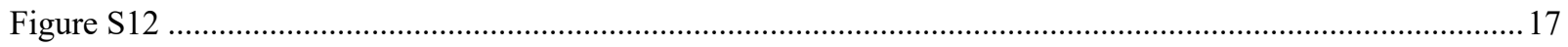

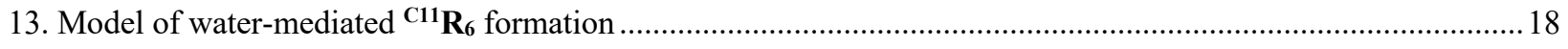

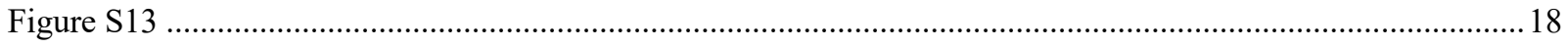

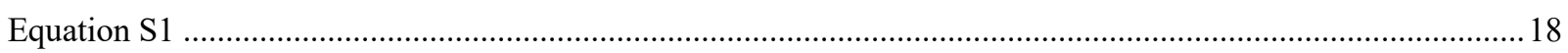

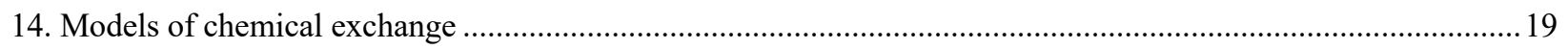

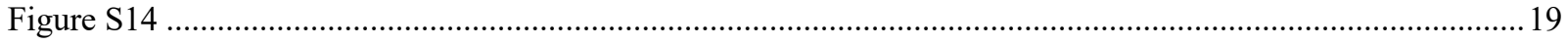

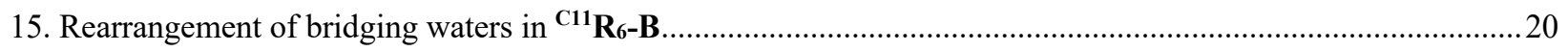

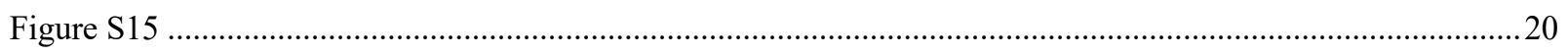

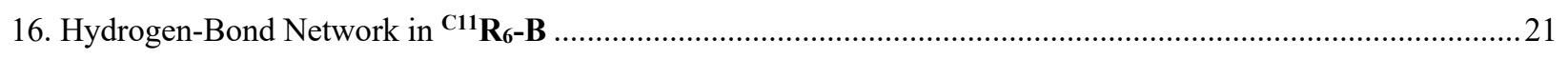

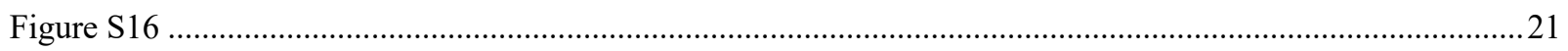

17. Computational Modelling of Sorbyl Alcohol Isomerization...............................................................22

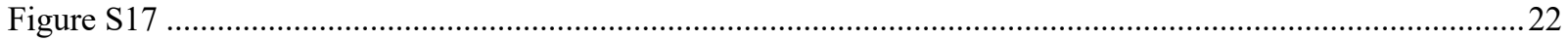




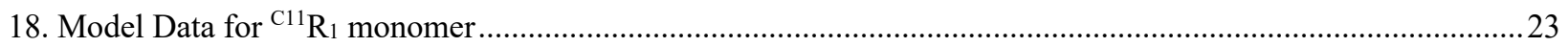

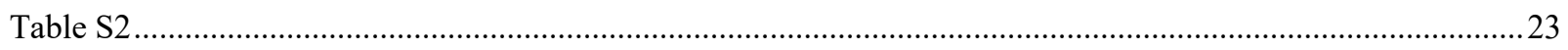

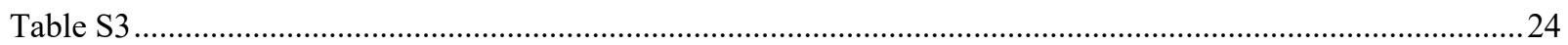

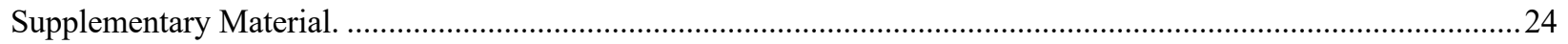

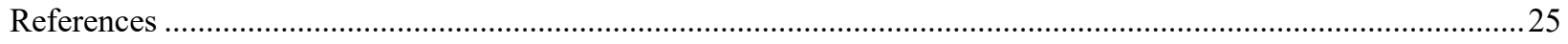




\section{Forcefield parameterization of resorcin[4] arene structures}

Model structures for hexameric methyl-resorcin[4]arene ( $\left.{ }^{\mathrm{C}_{1}} \mathbf{R}_{\mathbf{6}}\right)$ and undecyl-resorcin[4]arene $\left({ }^{\mathrm{C}_{11}} \mathbf{R}_{\mathbf{6}}\right)$ capsules were constructed, inclusive of 8 structural water molecules and minimized by DFT at a B3LYP/def2SVP level of theory with Gaussian 16 Rev C. ${ }^{1}$ Atom types, following GAFF2, and charge assignments, by RESP method, were made for the output structures using antechamber. ${ }^{2}$ The optimized ${ }^{{ }^{1}} \mathbf{R}_{\mathbf{6}}$ model served as a starting point for a short molecular dynamics simulation using xtb at a GFN2-xTB ${ }^{3}$ level of theory and implicit chloroform solvation. ${ }^{4} \mathrm{~A}$ total of 200 frames were extracted from the trajectory by random selection and single point energies were computed for these structures using DFT at a B3LYP/def2SVP level of theory using Gaussian 16 Rev C. ${ }^{1}$ A sample of 100 structures and single point energies were used to fit bond, angle, and torsion parameters from randomly generated values using paramfit. ${ }^{5}$ The remaining 100 structures and single point energies were used to validate the accuracy of the resulting forcefield in reproducing B3LYP/def2SVP energies in comparison to other common techniques as shown in Figure S1.
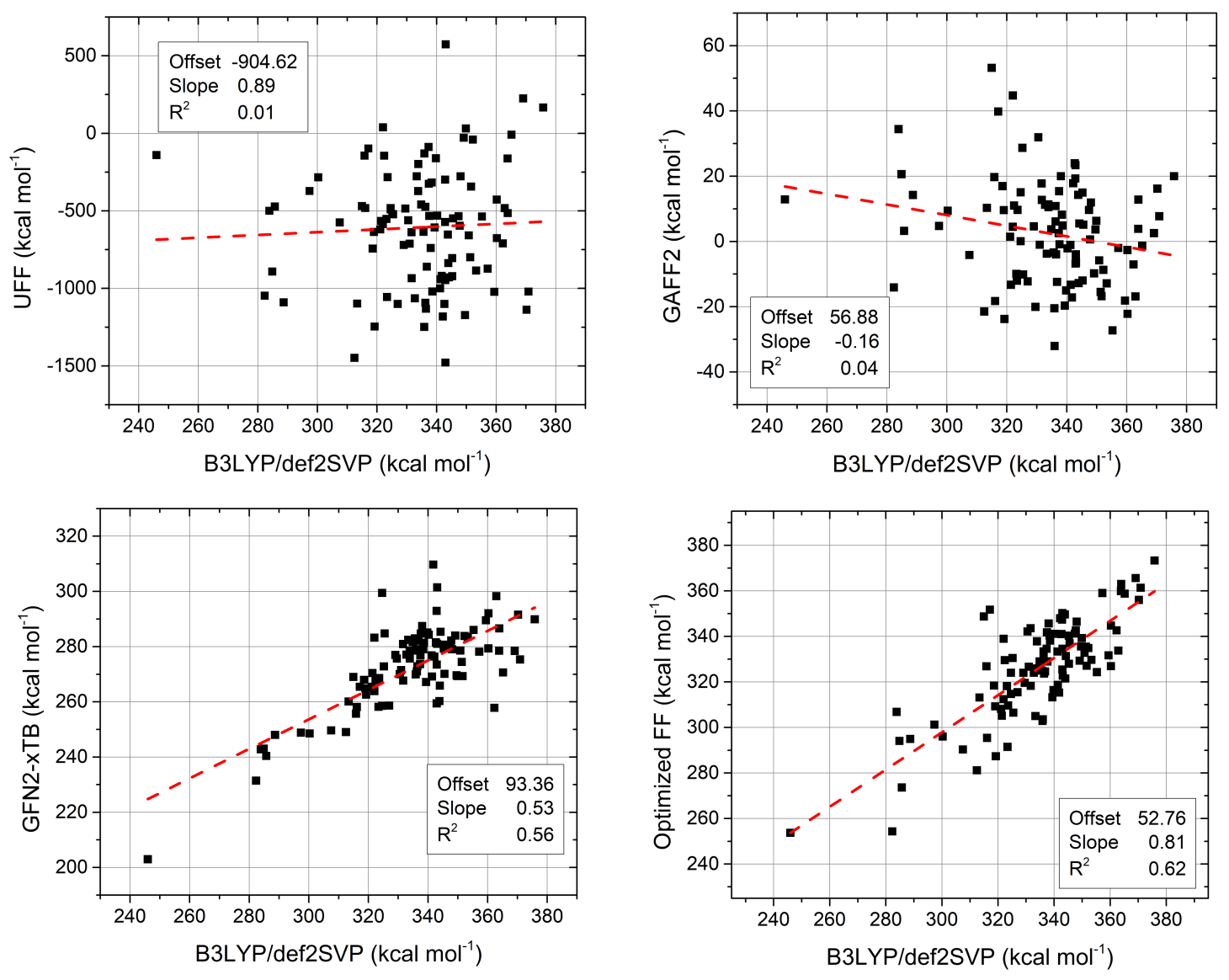

Figure S1. Comparison of optimized hexameric resorcin[4]arene capsule forcefield to common classical mechanics and semi-empirical techniques.

The genetically optimized parameter set demonstrated significant improvements over generalized forcefield methods, $\mathrm{GAFF}^{6}{ }^{6}$ and $\mathrm{UFF}^{7}$, and performed comparably to GFN2-xTB ${ }^{3}$ in reproducing DFT energies.

Modelling the complete ${ }^{{ }^{C 11}} \mathbf{R}_{6}$ capsule required additional parameters for the pendant undecyl groups, the optimization of which would be computationally expensive. Instead, these parameters were obtained from the GAFF2 forcefield which is well developed for simple $\mathrm{sp}^{3}$ hydrocarbons. ${ }^{6}$ 


\section{General procedures for CMD simulations}

Simulation environments were prepared using packmol, ${ }^{8}$ and contained a single ${ }^{\mathbf{C 1} 1} \mathbf{R}_{\mathbf{6}}$ capsule with $8-24$ SPC-flexible water molecules, ${ }^{9}$ and 1000 chloroform molecules in a 35 angstrom cube. In all simulations 8 water molecules were specifically placed at the vertex positions of the ${ }^{\mathbf{C} 11} \mathbf{R}_{\mathbf{6}}$ capsule. Classical mechanics dynamics simulations were carried using pmemd.CUDA ${ }^{10,11}$ following standard equilibration and productive phases indicated by the input files shown below in Table S1.

Table S1. Control input files for molecular dynamics simulations used in computational investigations used in a standard sequence of minimization, heating, density equilibration, thermal equilibration and productive molecular dynamics. Thermal equilibration and productive molecular dynamics used the same input file with appropriate simulation lengths (listed here as '\#\#\#').

\begin{tabular}{|c|c|}
\hline 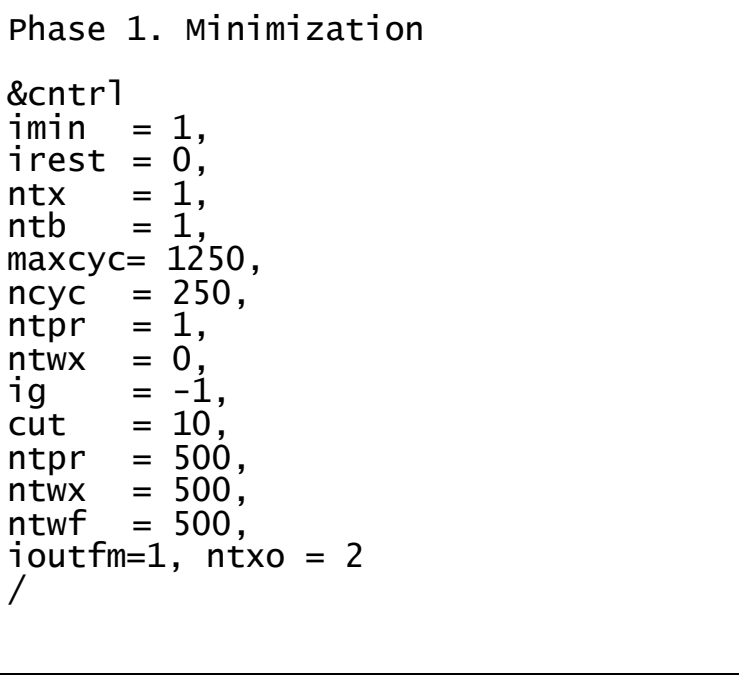 & 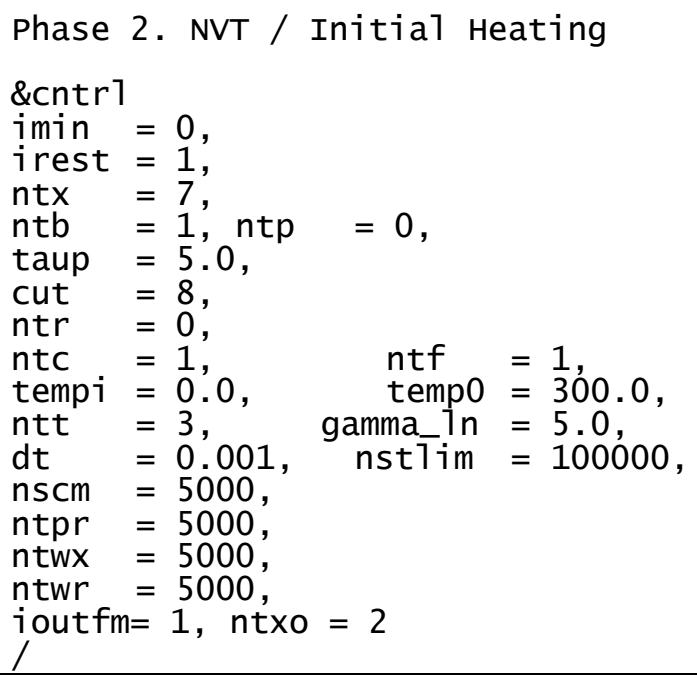 \\
\hline 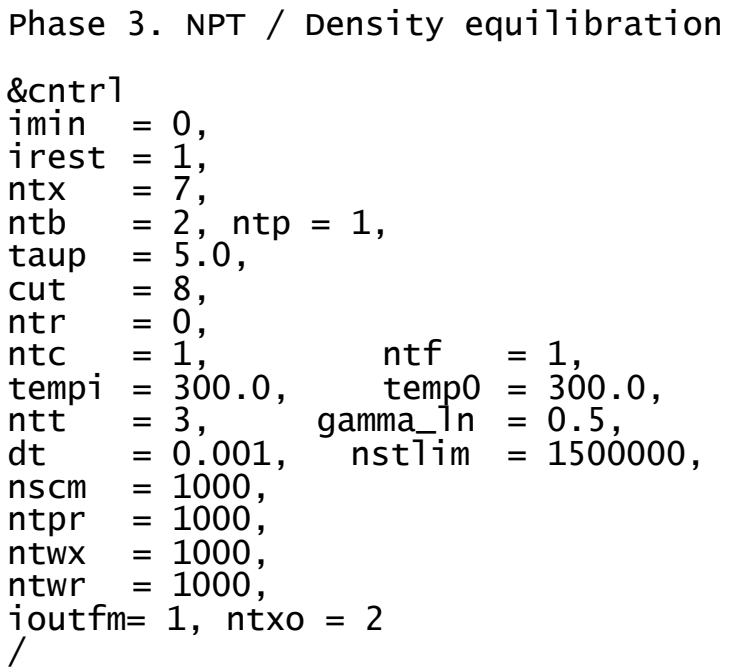 & 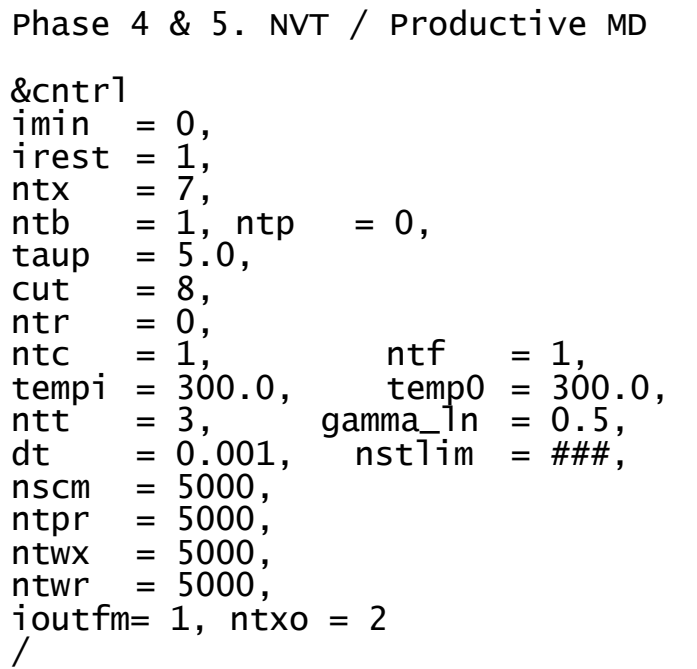 \\
\hline
\end{tabular}




\section{CMD studies of interfacial hydrogen bonding}

A total of 550 simulations were constructed containing 9-24 water molecules in order to observe the association of water and its effect on hydrogen bonding between the phenolic functional groups of adjacent resorcin[4]arene facial monomers. These simulations were propagated for $20 \mathrm{~ns}$ of productive molecular dynamics, and the resulting trajectories analyzed to compute hydrogen bonding between the resorcin[4]arene monomers as shown in Figure S2. A large number of trajectories were used in order to mitigate potential bias.

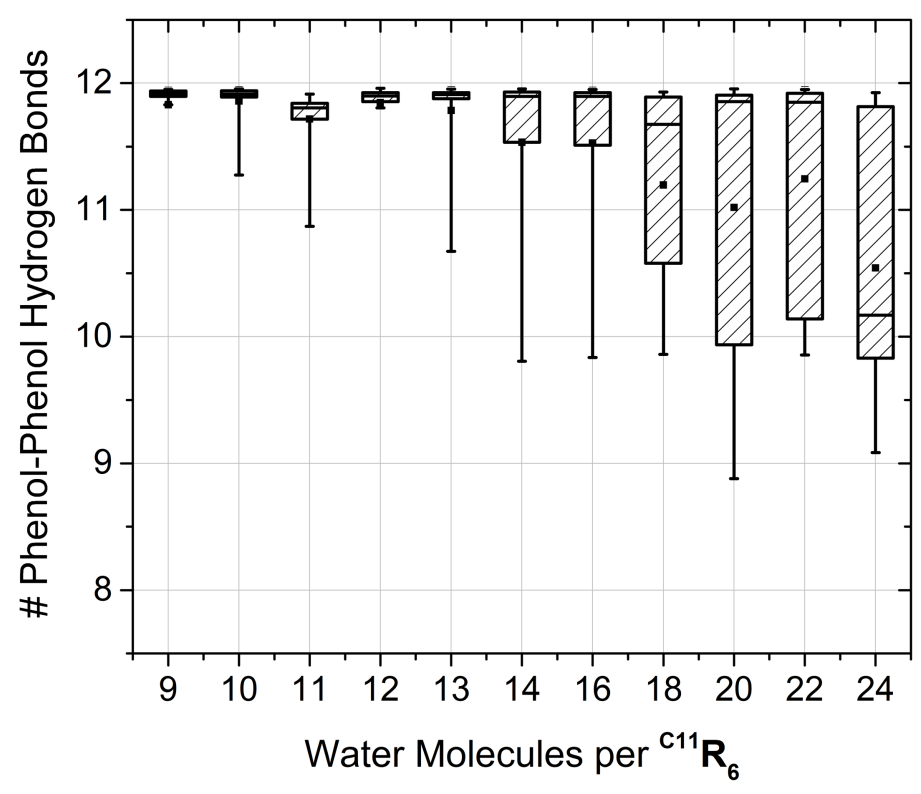

Figure S2. Hydrogen bond analysis results. The number of hydrogen bonds between resorcin[4]arene monomers were determined by standard analysis procedures implemented in cpptraj. ${ }^{10}$

We observed a propensity that simulations with $\geq 14$ water molecules resulted a disruption of interfacial hydrogen bonds, corresponding to the opening of 1-2 edges of the capsule's cubic structure as depicted in Figure 2. This change fundamentally alters the structure, resulting in an 'open state' distinct from typical portrayals or structural models of the 'closed state' capsule.

\section{GIST thermodynamic analysis of water association}

A set of 28 molecular dynamics simulations were constructed featuring 8-22 waters. Trajectories from these simulations were propagated for $800 \mathrm{~ns}$ each to sample the distribution of water within the capsule environment (Table S1). These trajectories were then analyzed using grid inhomogeneous solvation theory (GIST) to estimate the thermodynamics of water association. ${ }^{12}$ The energy per water molecule was determined from the volume sum of the entropic and enthalpic components divided among the water molecules. The relative energy compared to 8 water molecules per capsule then signifies the thermodynamics of water association, as illustrated in Figure 2. 


\section{Materials}

Resorcinol was purchased from Sigma Aldrich; darker crystals were removed manually during weighing as a means of purification before use. Decanal was similarly purchased from Sigma and used immediately after receipt without any purification. Ethanol, concentrated aqueous $\mathrm{HCl}$, methanol, and acetone were purchased from VWR and used directly without purification. All NMR samples were prepared in $\mathrm{CDCl}_{3}$, purchased from Sigma Aldrich and filtered through a column of dry activated basic alumina before use.

\section{Analysis methods}

${ }^{1} \mathrm{H}$ and ${ }^{31} \mathrm{P}$ spectra were measured on a Bruker ARX 300 at a constant temperature of $298 \mathrm{~K}$. Spectral data were referenced to residual solvent protons $\left(7.26 \mathrm{ppm}\right.$ for $\left.\mathrm{CDCl}_{3}\right)$ for ${ }^{1} \mathrm{H}$ measurements, or the internal deuterium lock signal for ${ }^{31} \mathrm{P}$ measurements

Karl-Fisher titrations were carried out using a Metrohm 831 KF Coulometer system.

\section{Synthesis of undecyl-resorcin[4]arene $\left({ }^{\mathrm{C} 11} \mathbf{R}_{1}\right)$}

The synthesis of the title compound was carried out with slight modification from reported procedures ${ }^{13} \mathrm{~A}$ round bottom flask was charged with resorcinol $(20.0 \mathrm{~g}, 90.8 \mathrm{mmol})$ dissolved in a mixture of ethanol $(30 \mathrm{~mL})$ and concentrated aqueous hydrochloric acid $(15 \mathrm{~mL})$. The flask was then cooled in an ice bath and solution of dodecanal $(15.3 \mathrm{~g}, 18.0 \mathrm{~mL}, 89.6 \mathrm{mmol})$ in ethanol $(15 \mathrm{~mL})$ was added dropwise by an addition funnel over approximately 1 hour. The ice bath was removed and the mixture was heated $\left(50^{\circ} \mathrm{C}\right)$ while stirring overnight $(20 \mathrm{~h})$. Over time a yellow to red color developed in the solution, and the next morning an off-white precipitate was observed. This precipitate was collected by vacuum filtration and washed with a solution of saturated aqueous sodium bicarbonate $(50 \mathrm{~mL})$ to neutralize remaining acid, and then demineralized water $(3 \times 50 \mathrm{~mL})$ to remove any remaining bicarbonate. The filtrate was dissolved in hot acetone (ca. $200 \mathrm{~mL}$ ) and upon cooling the near-white precipitated solid was collected and washed with cold acetone until the washings were colorless. The filtrate was then dissolved and precipitated upon cooling from hot methanol to obtain the final product as a white solid $(9.0 \mathrm{~g}, 38 \%$ yield).

\section{Assembly of hexameric undecyl-resorcin [4] arene capsules $\left({ }^{\mathrm{C} 11} \mathbf{R}_{6}\right)$}

A suspension of ${ }^{\mathbf{C} 11} \mathbf{R}_{\mathbf{1}}(37.5 \mathrm{mg}, 34.1 \mu \mathrm{Mol})$ in $\mathrm{CDCl}_{3}(1 \mathrm{~mL})$ was produced in a screw cap vial $(5 \mathrm{~mL})$. The vial was held above a beaker of hot water $\left(60-70^{\circ} \mathrm{C}\right)$ and mixed with magnetic stirring. We found that the steam provided from the hot water bath afforded sufficient moisture for capsule formation and prevented the addition of excessive water to the solution which may later phase-separate.

\section{Preparation of ${ }^{\mathrm{C} 11} \mathbf{R}_{6}$ samples with varied water content}

Samples of ${ }^{{ }^{11}} \mathbf{R}_{\mathbf{6}}$ were prepared and an addition of water $(2 \mathrm{uL}, 110 \mu \mathrm{Mol})$ was added by micropipette. A small quantity (ca. 5-6 pieces) of molecular sieves (3.0 $\AA$ ) were added to the sample vial with a small Teflon stir bar. The sample was stirred for up to 3 hours to remove water before being filtered into an NMR tube for analysis. Quantitation by ${ }^{1} \mathrm{H}$ NMR spectroscopy, shown in Figure S5, indicates minimal changes aside water content and phenolic peak distribution, indicating that ${ }^{{ }^{\mathbf{1}} \mathbf{R}_{\mathbf{6}}}$ is chemically stable under these conditions.

A separate sample was prepared for DOSY NMR following identical protocols. Using the auto-diffusion of residual $\mathrm{CHCl}_{3}$ to correct for changes in solvent viscosity, we estimate the Stokes radii of ${ }^{\mathrm{C} 11} \mathbf{R}_{\mathbf{6}}$ to be $22 \AA$ for both assemblies, which is reasonable for the hexameric species.

Transmission FTIR was then used to obtain vibrational spectra of ${ }^{\mathbf{C} 11} \mathbf{R}_{\mathbf{6}}$ before and after the drying process to specifically identify potential chemical changes, or the formation of dimeric $\left({ }^{\mathbf{C} 11} \mathbf{R}_{\mathbf{2}}\right)$ species which have been previously analyzed by this method. ${ }^{14}$ 

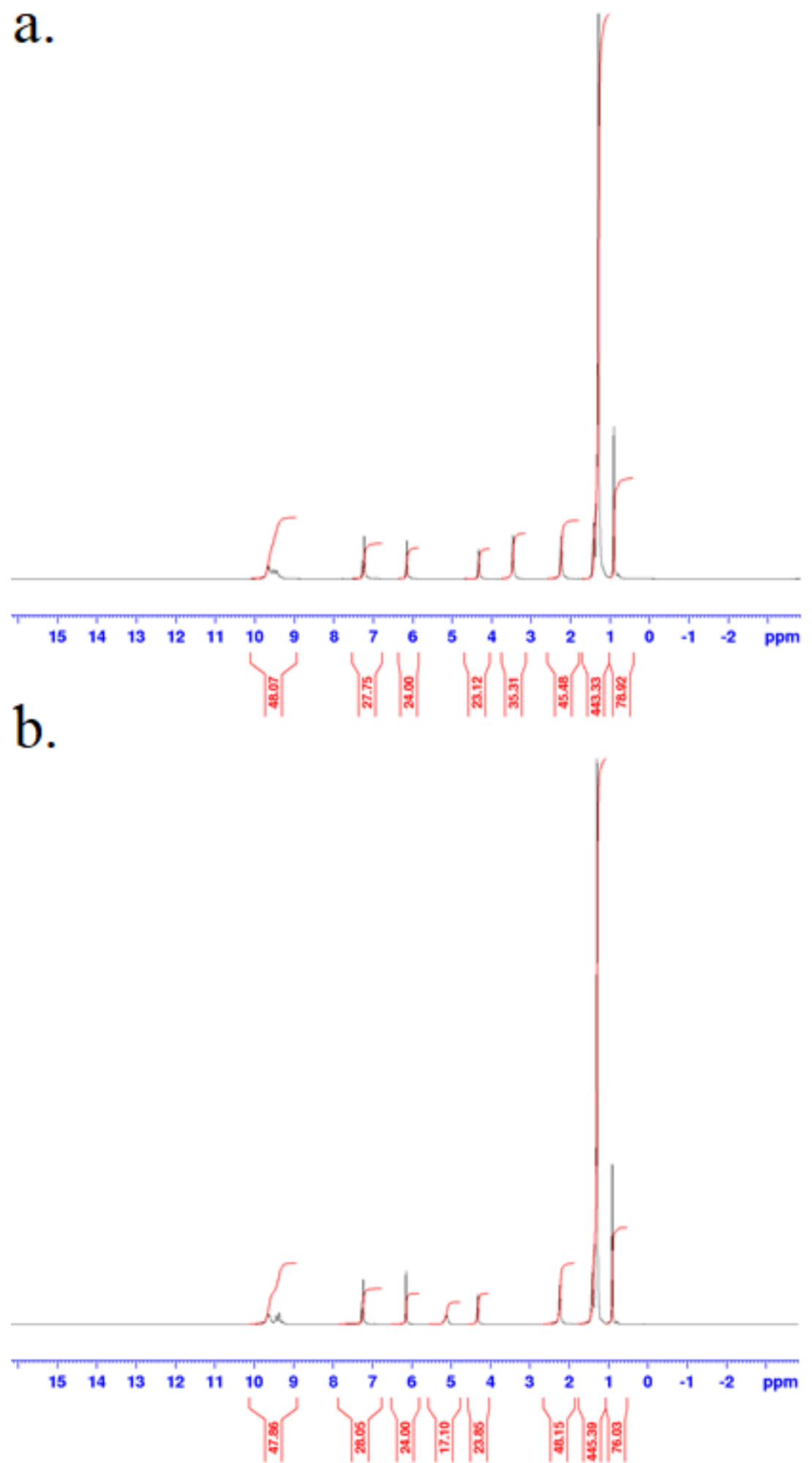

Figure S3. ${ }^{1} \mathrm{H} N \mathrm{NR}\left(500 \mathrm{MHz}, \mathrm{CDCl}_{3}\right)$ spectra obtained from ${ }^{\mathrm{C11}} \boldsymbol{R}_{6}$ as prepared (a) and after drying (b). 


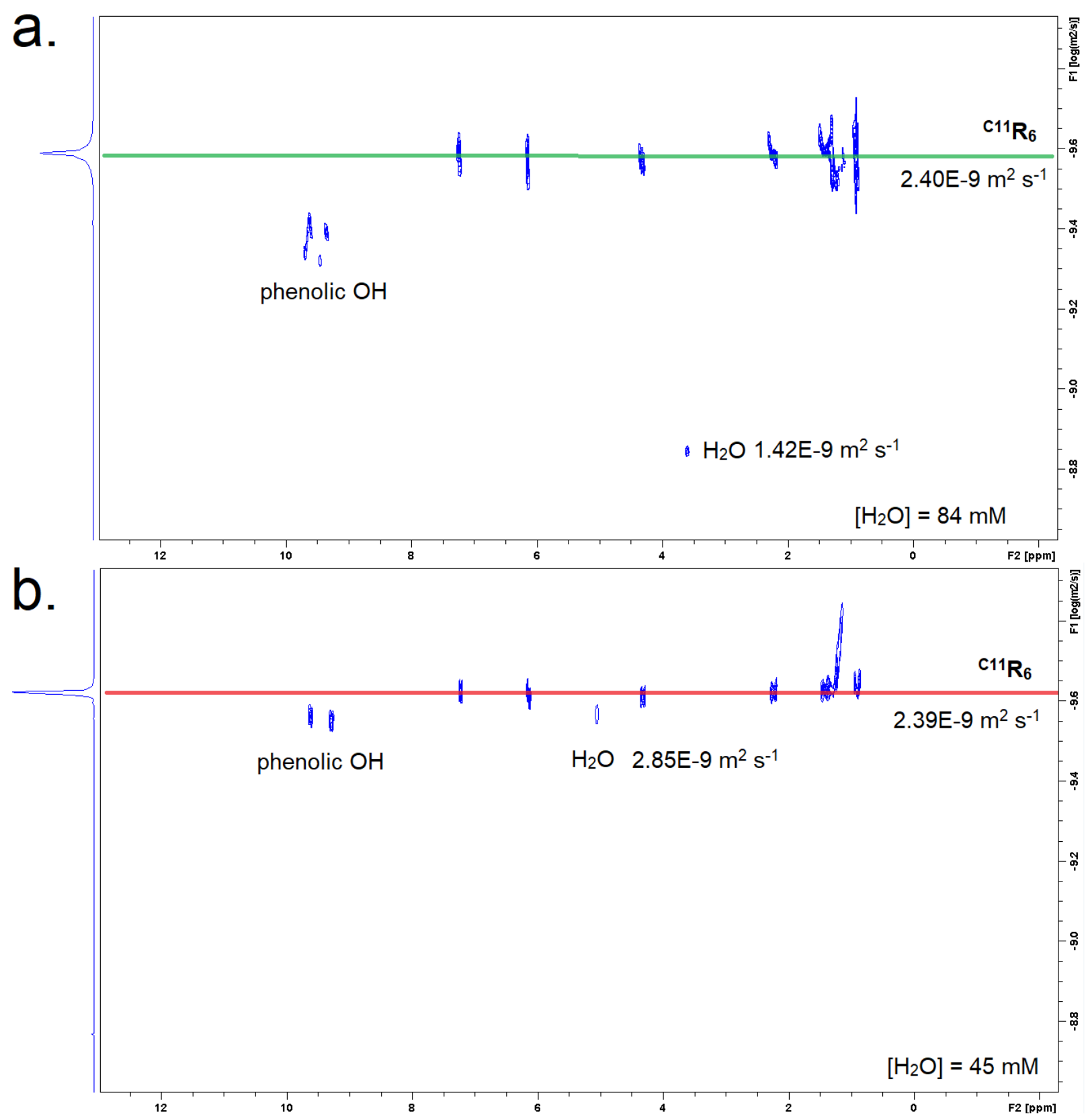

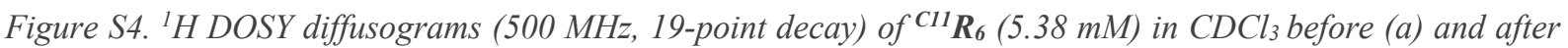
(b) drying by molecular sieves. Diffusion was determined by exponential fit using Topspin 4.0.9 "DOSY $2 d$ " automation program with a 2-component fitting of the ${ }^{1} \mathrm{H}$ spectra obtained at 19 gradient strengths using a standard LED-type pulse program (ledbpgb2s). 


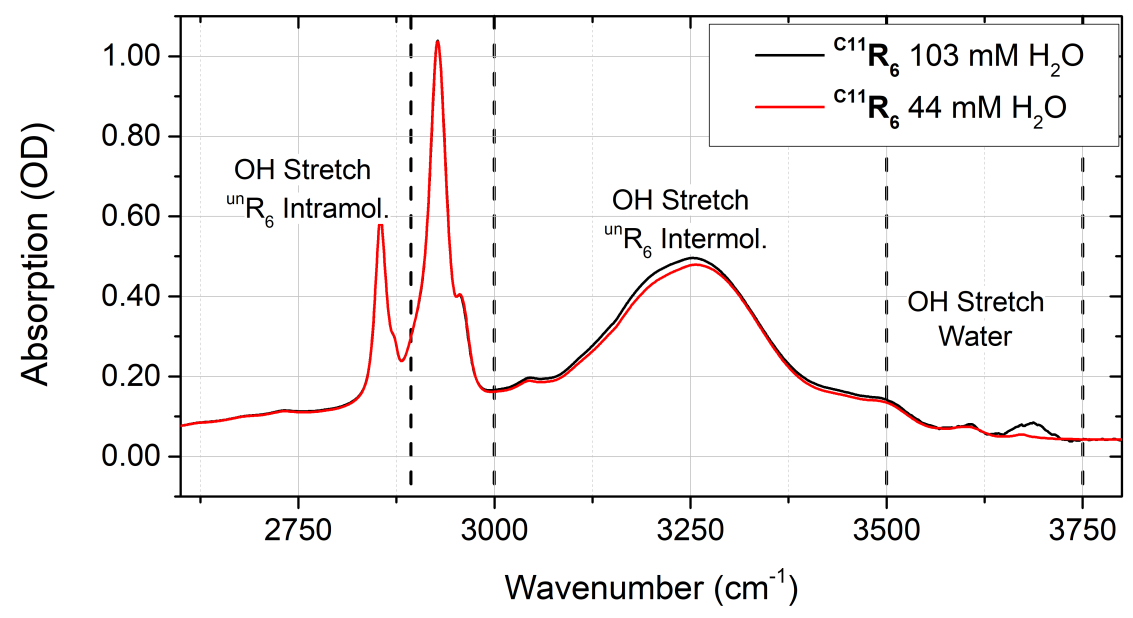

Figure S5. FTIR Spectra (KBr cell, $100 \mu \mathrm{m})$ obtained from solution state samples of ${ }^{C 11} \boldsymbol{R}_{6}$ in $\mathrm{CDCl}_{3}$ as prepared (103.12 $\left.\mathrm{mM} \mathrm{H}_{2} \mathrm{O}\right)$, and after drying by molecule sieves (44.03 $\left.\mathrm{mM} \mathrm{H}_{2} \mathrm{O}\right)$.

These spectral features match previously reported spectra of the hexameric hexyl resorcin[4]arene capsules and not their dimeric analogs which feature a sharp band at $3500 \mathrm{~cm}^{-1} .{ }^{14}$ Therefore we conclude that despite changes in size, ${ }^{\mathbf{C} 11} \mathbf{R}_{6}$ remains a hexamer in $\mathrm{CDCl}_{3}$ containing between $44-103 \mathrm{mM}$ water. 


\section{0. ${ }^{\mathrm{C} 11} \mathbf{R}_{6}$ catalyzed Diels-Alder cycloaddition}

Solutions of sorbic alcohol $(24 \mathrm{mM})$ and maleimide $(24 \mathrm{mM})$ were prepared in $\mathrm{CDCl}_{3} / 0.1 \%$ TMS and dried using molecular sieves $(3 \AA)$. Additionally, a series of ${ }^{{ }^{11}} \mathbf{R}_{6}$ solutions were prepared and dried following the above procedures in $\mathrm{CDCl}_{3} / 0.1 \%$ TMS to afford samples of the capsule in the presence of 48 to $130 \mathrm{mM}$ of water as determined by automated Karl Fischer titration. A catalytic experiment was conducted by the addition of the sorbic alcohol solution ( $24 \mathrm{mM}, 125 \mu \mathrm{L}), \mathrm{CDCl}_{3} / 0.1 \%$ TMS $(125 \mu \mathrm{L})$, maleimide solution $(24 \mathrm{mM}, 125 \mu \mathrm{L})$ and a ${ }^{{ }^{C 11} \mathbf{R}_{6}}$ solution to a dry NMR tube by micropipette. This sample was vortexed briefly (ca. 3 seconds) and inserted immediately into the NMR for analysis where ten ${ }^{1} \mathrm{H}$ NMR spectra (300 MHz, $\mathrm{T}_{\mathrm{r}}=25$ seconds) were obtained over 40 minutes. The NMR sample chamber as well as the laboratory environment were temperature controlled to $25^{\circ} \mathrm{C}$. The depletion of maleimide ( $\delta=6.72 \mathrm{ppm})$ was computed with reference to the internal TMS standard. A linear fit was used to determine the initial reaction velocity as shown in Figure S7. Slight variation was apparent in the initial concentration of maleimide, likely due to differences in timing from sample mixing to the completion of the first measurement.

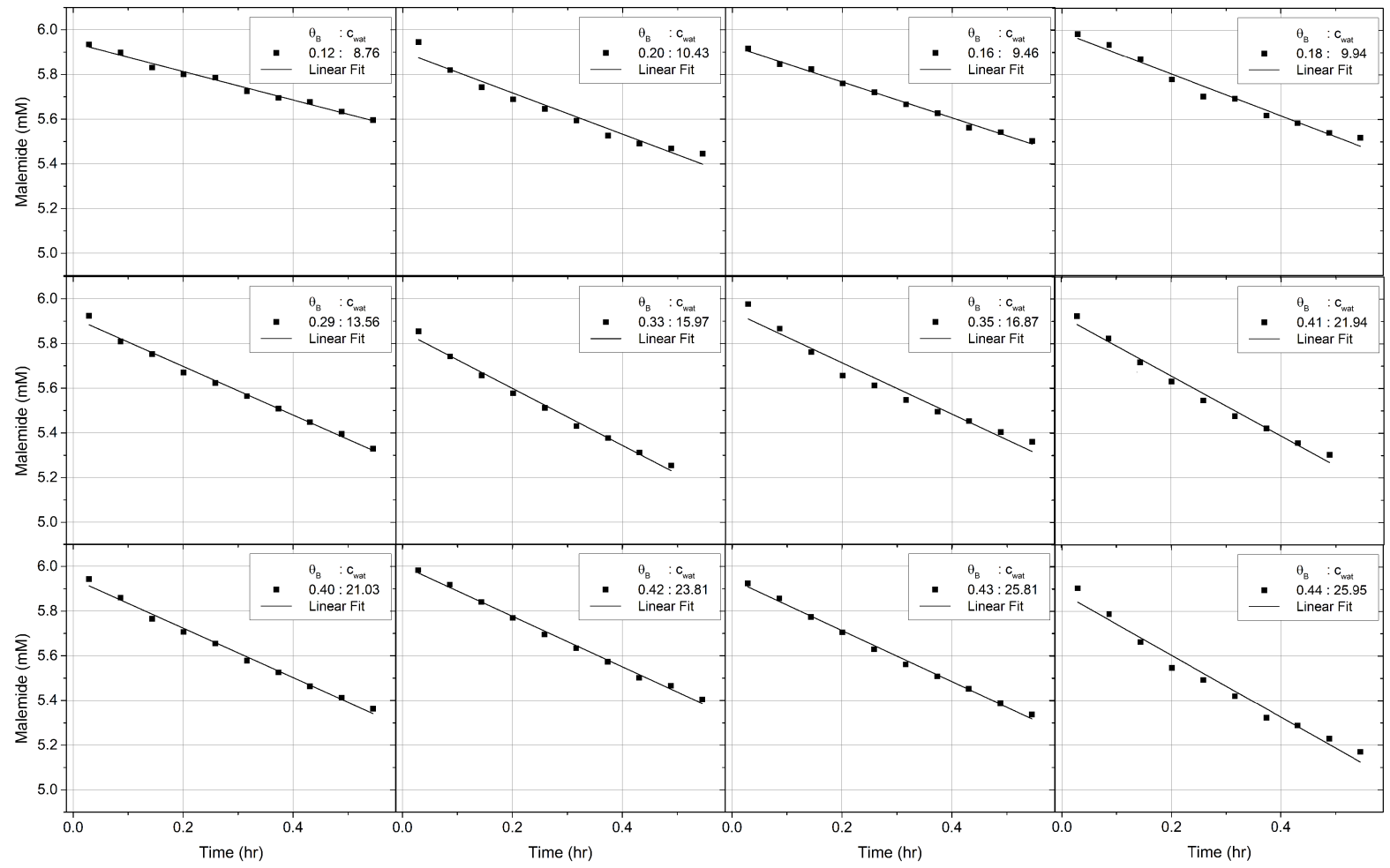

Figure S6. Maleimide depletion observed by ${ }^{1} H$ NMR for a ${ }^{C 11} \boldsymbol{R}_{6}$ catalyzed Diels-Alder cycloaddition reaction at water contents $\left(c_{\text {wat }}\right)$ spanning 8.76-25.95 mM, shown with linear fits used to determine the reaction rate. 


\section{Uncatalyzed Diels-Alder cycloaddition:}

Solutions of sorbic alcohol $(24 \mathrm{mM})$ and maleimide $(24 \mathrm{mM})$ were prepared in $\mathrm{CDCl}_{3} / 0.1 \%$ TMS and dried using molecular sieves ( $3 \AA)$. A reaction was prepared by the sequential addition of the sorbic alcohol solution $(125 \mu \mathrm{L})$ and maleimide solution $\left(125 \mu \mathrm{L}\right.$ ) to either dry or water-saturated $\mathrm{CDCl}_{3} / 0.1 \% \mathrm{TMS}$, followed by brief vortexing (ca. 3 seconds). The samples were periodically analyzed by ${ }^{1} \mathrm{H}$ NMR $\left(300 \mathrm{MHz}, \mathrm{T}_{\mathrm{r}}=25 \mathrm{~s}\right)$ overnight, and the depletion of maleimide $(\delta=6.72 \mathrm{ppm})$ was determined with reference to the internal TMS standard. A linear fit was used to estimate the reaction velocity from data obtained over the first 5 hours as shown in Figure S8. The reaction rate of the water-saturated or 'wet' reaction was found to be $0.21 \pm 0.01 \mathrm{mM} \mathrm{hr}^{-1}$, ca. $31 \%$ faster than the rate observed for the reaction in the absence of water, $0.16 \pm 0.01 \mathrm{mM} \mathrm{hr}^{-1}$. The observed rates are significantly slower than the ${ }^{{ }^{C 11} \mathbf{R}_{6}}$ catalyzed reactions $\left(0.6-1.1 \mathrm{mM} \mathrm{hr}^{-1}\right)$, and the effect of water upon the rates is significantly less in these uncatalyzed reactions (ca. $31 \%$ vs. ca. $182 \%$ increase).

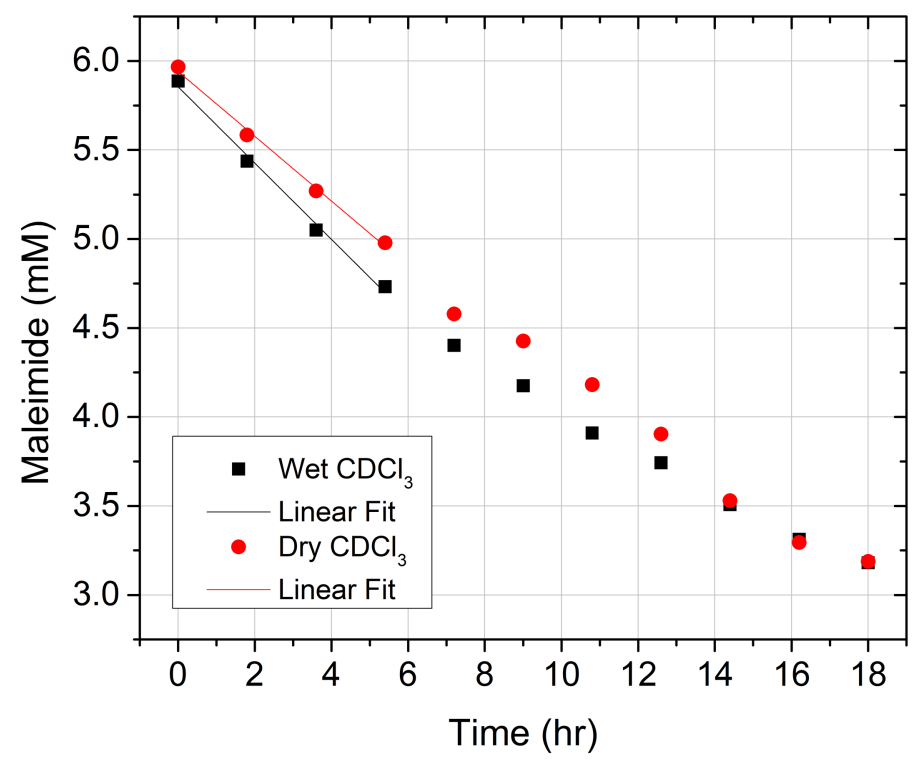

Figure S7. Maleimide depletion observed by ${ }^{l} H$ NMR for the uncatalyzed Diels-Alder cycloaddition reaction in ordinary $\mathrm{CDCl}_{3}$ ('dry', red), and 50\% volume water-saturated $\mathrm{CDCl}_{3}$ ('wet', black). Linear fits on the initial 5 hours of the reaction time were computed to determine the initial reaction velocity. 


\section{2. ${ }^{31} P$ NMR studies of ${ }^{C 11} R_{6}$ internal acidity}

Two samples of ${ }^{{ }^{C 11}} \mathbf{R}_{\mathbf{6}}(2 \mathrm{~mL}, 5.38 \mathrm{mM})$ were prepared as described above in $\mathrm{CDCl}_{3}$. To each of these samples a quantity of solid $\mathrm{Bu}_{3} \mathrm{PO}$ was added (either $1.52 \mathrm{mg}$ or $8.73 \mathrm{mg}$ ) to produce samples with 0.65 or 3.72 equivalents of guest. These solutions were divided into two separate aliquots, one which was dried by molecular sieves (3.0 $\AA$ ), and the other, 'wet' solution, remained with its initial water content. These solutions were then analyzed directly by ${ }^{1} \mathrm{H}$ $\operatorname{NMR}\left(\mathrm{T}_{\mathrm{r}}=1 \mathrm{~s}, 300 \mathrm{MHz}\right)$, and ${ }^{31} \mathrm{P}$ NMR $\left({ }^{1} \mathrm{H}\right.$ decoupled, $\left.121.44 \mathrm{MHz}, \mathrm{T}_{\mathrm{r}}=0.2 \mathrm{~s}, \mathrm{~ns}=256\right)$ as shown in Figures $\mathrm{S} 10$, $\mathrm{S} 11, \& \mathrm{~S} 12$. The dried solutions were then titrated with the 'wet' solutions to observe ${ }^{31} \mathrm{P}$ chemical shifts of the encapsulated and free species over a range of water contents. From the resulting ${ }^{1} \mathrm{H}$ spectra, the integrated area of the water peak relative to ${ }^{{ }^{C 11}} \mathbf{R}_{\mathbf{6}}(\delta=6.22 \mathrm{ppm}, 24 \mathrm{H})$ was used to determine water content assuming a stable ${ }^{{ }^{\mathrm{C} 11}} \mathbf{R}_{\mathbf{6}}$ concentration $(5.38 \mathrm{mM})$. After titration was complete, the sample featuring $24.00 \mathrm{mM}$ of $\mathrm{Bu}_{3} \mathrm{PO}$ guest was analyzed by ${ }^{1} \mathrm{H}_{-}{ }^{31} \mathrm{P}$ HMBC to assist peak assignment (Figure S13), DOSY NMR to validate capsule integrity and identify peaks of the encapsulated $\mathrm{Bu}_{3} \mathrm{PO}$ by ${ }^{1} \mathrm{H}$ NMR (Figure S14).

In the dried sample (44.34 mM Water) containing the lower concentration of $\mathrm{Bu}_{3} \mathrm{PO}(3.50 \mathrm{mM})$, we observed the near total $(95.90 \%)$ encapsulation of $\mathrm{Bu}_{3} \mathrm{PO}$ by ${ }^{1} \mathrm{H}$ NMR, resulting in a remarkably sharp NMR spectrum (Figure S10). Upfield peaks $(\delta-2.0-0.5)$ correspond to the ${ }^{31} \mathrm{P}$ coupled protons of the encapsulated species.

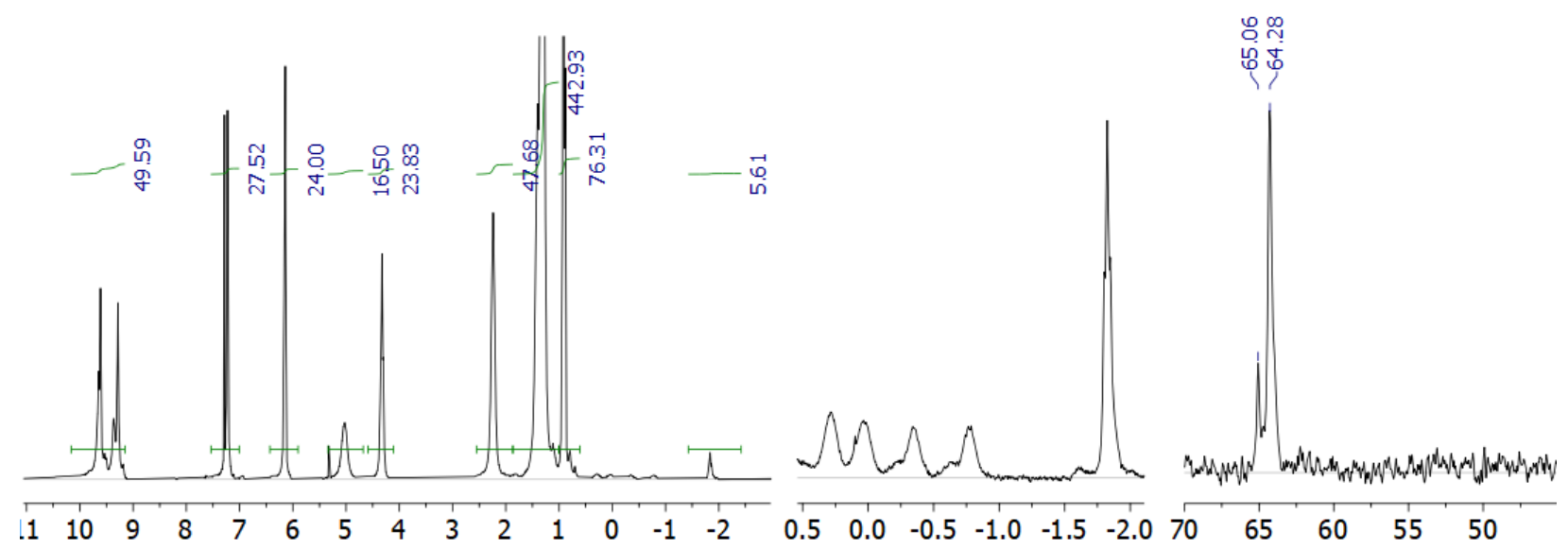

Figure S8. ${ }^{1} \mathrm{H}$ (left and middle), and ${ }^{31} \mathrm{P}$ (right) NMR spectra obtained for a sample of ${ }^{C 11} \boldsymbol{R}_{6}(5.38 \mathrm{mM})$ at low water content (44.18 mM) showing the encapsulation of Bu3PO (middle, $\delta=-2.0-0.5 \mathrm{ppm}$ ) and a single species appears in ${ }^{1} H$ decoupled ${ }_{31} P N M R$.

In these ${ }^{1} \mathrm{H}$ decoupled ${ }^{31} \mathrm{P}$ spectra, a major and minor peak are observed, we putatively assign this major peak $\left(\delta_{31 \mathrm{P}}=\right.$

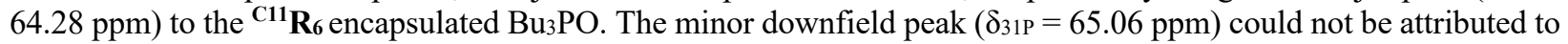
a particular species experimentally due to its low intensity and fast relaxation. This peak is observable in ${ }^{31} \mathrm{P}$ spectra at both high and low concentrations of $\mathrm{Bu}_{3} \mathrm{PO}$ (Figures $\mathrm{S} 11$ \& S12, respectively), decreasing in intensity with increasing water content.

During the titration of samples with a low $(3.50 \mathrm{mM})$ and high $(24.00 \mathrm{mM})$ concentrations of $\mathrm{Bu}_{3} \mathrm{PO}$ we observed a third peak which we assigned to the free $\mathrm{Bu}_{3} \mathrm{PO}$. Interestingly, in ${ }^{1} \mathrm{H}$ NMR we observe the decrease of encapsulated $\mathrm{Bu}_{3} \mathrm{PO}$ concomitant with increasing water content. We attribute this decrease of encapsulation to the favorable formation of hydrogen bonds between $\mathrm{Bu}_{3} \mathrm{PO}$ and free water in the non-polar medium. Given the relatively high concentration of water compared to $\mathrm{Bu}_{3} \mathrm{PO}$, we surmise that while free water may compete with ${ }^{{ }^{11}} \mathbf{R}_{6}$ for $\mathrm{Bu}_{3} \mathrm{PO}$ association, free $\mathrm{Bu}_{3} \mathrm{PO}$ is unlikely to compete with ${ }^{\mathbf{C} 11} \mathbf{R}_{\mathbf{6}}$ for the association of the large excesses of free water. However, the effect of competition by free water for hydrogen bonded guests is interesting, and may impact catalytic applications - perhaps resulting in performance limitations as observed in this study (Figure 6). 


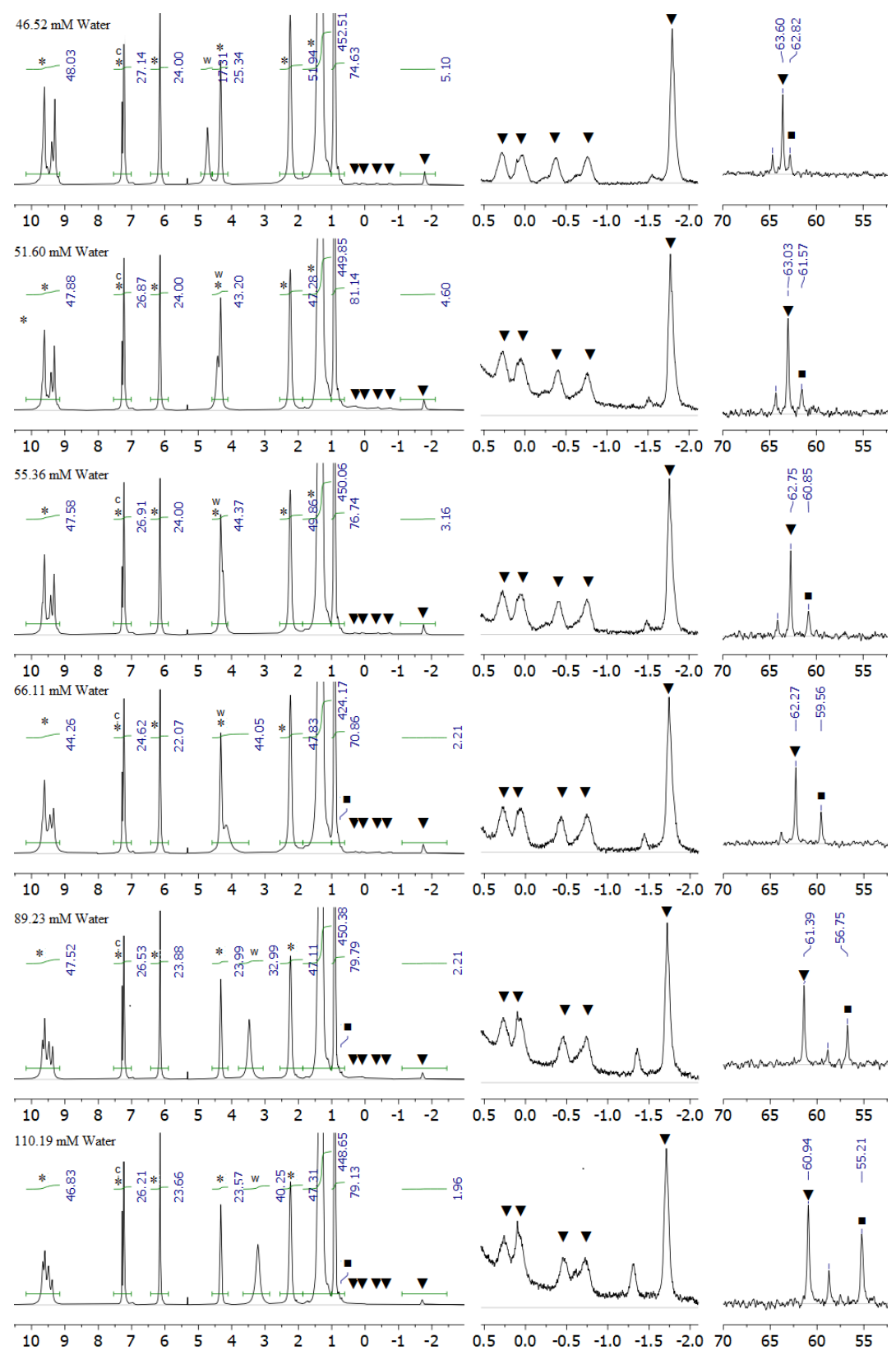

Figure S9. NMR spectra obtained for a solution of ${ }^{C 11} \boldsymbol{R}_{6}(5.38 \mathrm{mM})$ and $\mathrm{Bu}_{3} \mathrm{PO}(3.50 \mathrm{mM})$ in $\mathrm{CDCl}_{3}$ at water concentrations ranging from 46.52-110.19 mM as labeled. ${ }^{1} \mathrm{H} N M R$ spectra shows the capsule (left), with upfield shifted encapsulated Bu3PO (middle). Both free ( $\mathbf{\square})$ and encapsulated $(\boldsymbol{\nabla})$ Bu3PO were observed by both ${ }^{1} H N M R$ (left, middle) and ${ }^{31} \mathrm{P} N \mathrm{NM}$ (right). Peaks corresponding to ${ }^{C 11} \boldsymbol{R}_{6}\left({ }^{*}\right)$, water (w) and $\mathrm{CDCl}_{3}$ (c) are annotated for reference with overlapping peaks featuring double annotation (e.g., $c^{*}$ ). 


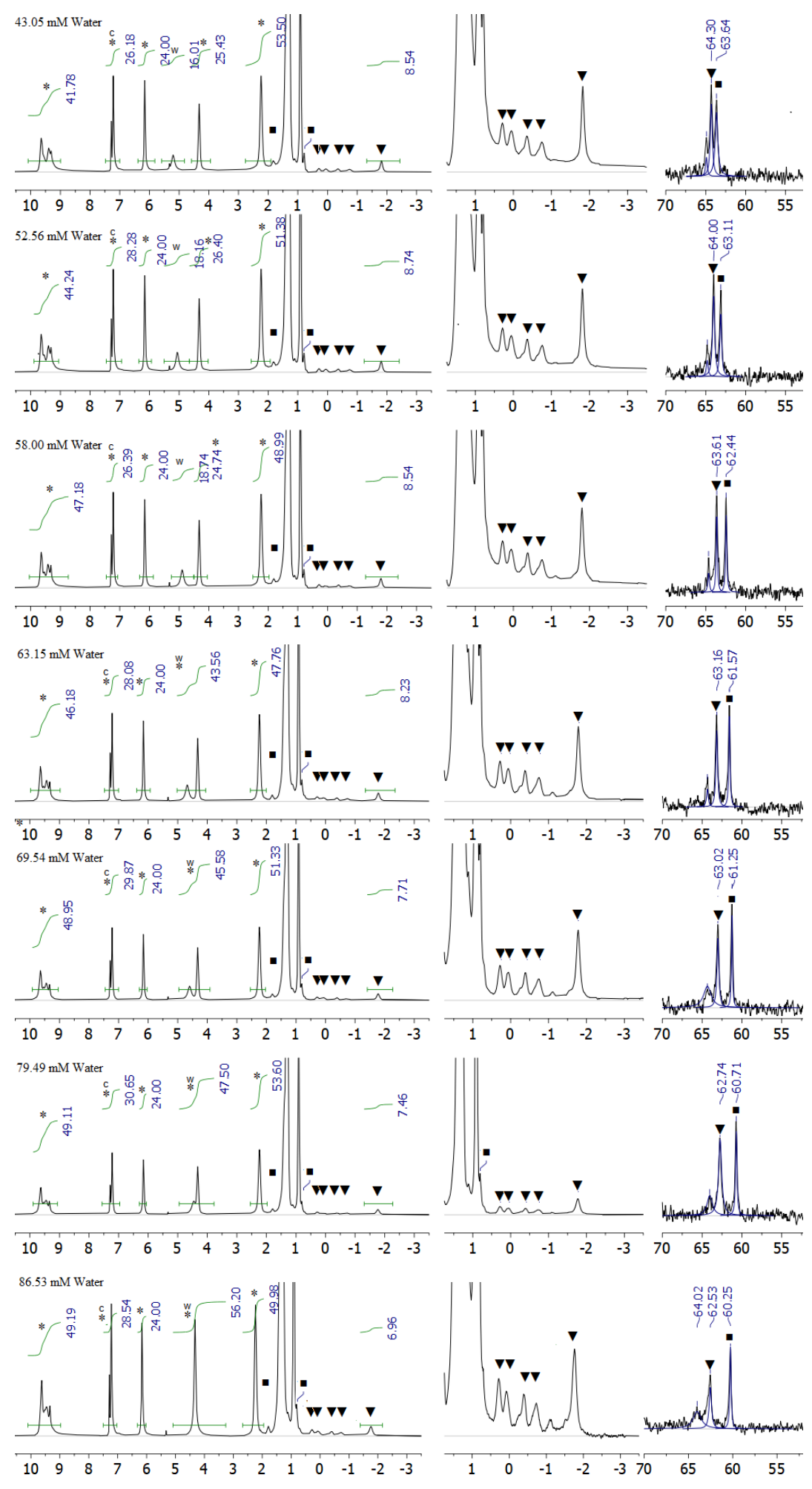

Figure S10. NMR spectra obtained for a solution of ${ }^{C 11} \boldsymbol{R}_{6}(5.38 \mathrm{mM})$ and $B u_{3} P O(24.00 \mathrm{mM})$ in $\mathrm{CDCl}_{3}$ at water concentrations ranging from 43.05-86.53 mM as labeled. ${ }^{1} \mathrm{H} N \mathrm{NR}$ spectra shows the capsule (left), with upfield shifted encapsulated Bu3PO (middle). Both free (-) and encapsulated ( $\mathbf{\nabla}) \mathrm{Bu}_{3} \mathrm{PO}$ were observed by both ${ }^{l} \mathrm{H} N \mathrm{NR}$ (left, middle) and ${ }^{31} \mathrm{P}$ NMR (right). Peaks corresponding to ${ }^{111} \boldsymbol{R}_{6}\left({ }^{*}\right)$, water (w) and $\mathrm{CDCl}_{3}(\mathrm{c}$ ) are annotated for reference with overlapping peaks featuring double annotation (e.g., $c^{*}$ ). 


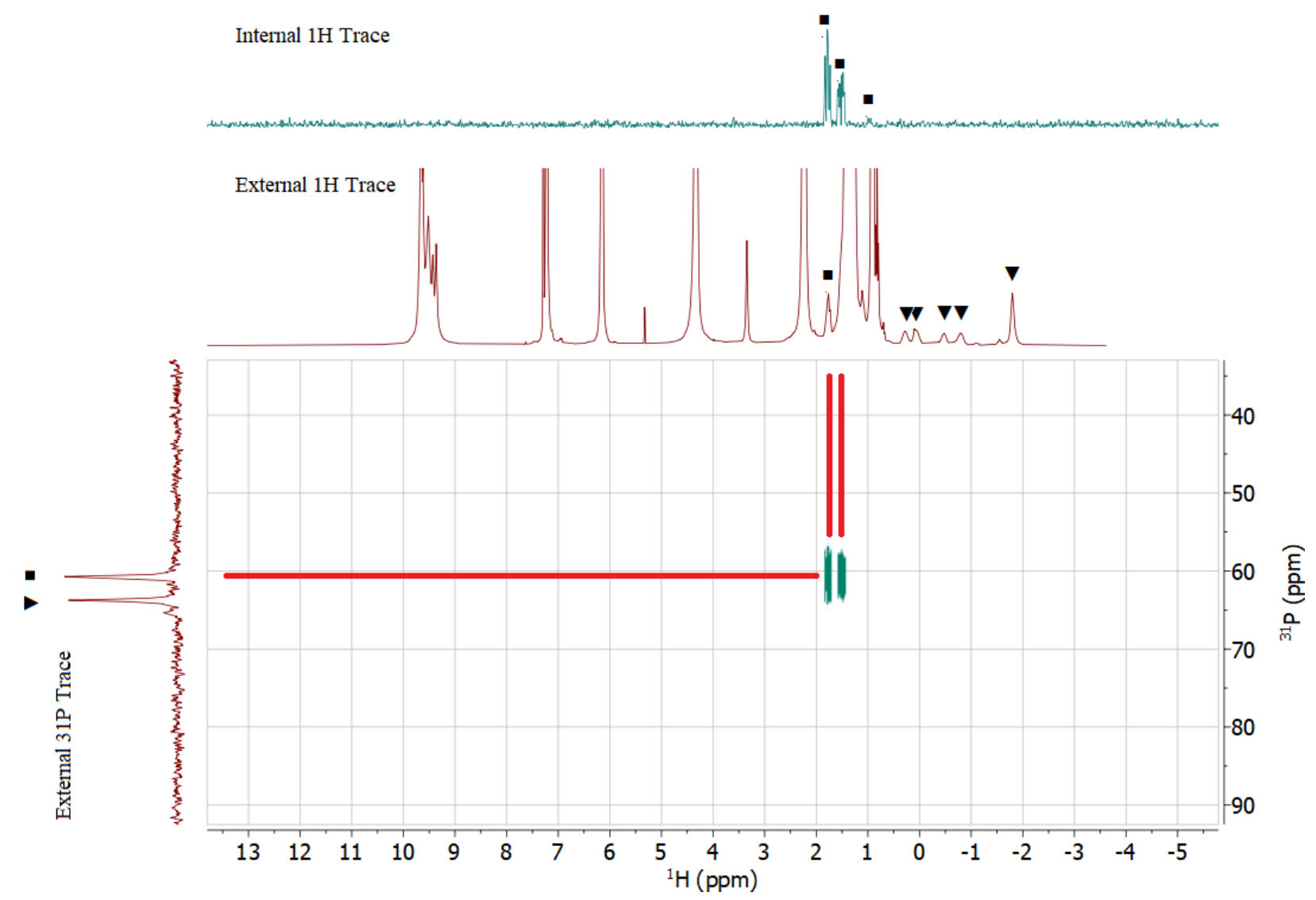

Figure S11. ${ }^{1} \mathrm{H}^{31}{ }^{3} \mathrm{P} \mathrm{HMBC}\left({ }^{1} \mathrm{H}=300 \mathrm{MHz}, \mathrm{ns}=5120, \mathrm{td}=64\right)$ spectra obtained for a sample of Bu $\mathrm{PO}(24.00$ $\mathrm{mM})$ and ${ }^{\mathrm{C} 11} \boldsymbol{R}_{\mathbf{6}}(5.38 \mathrm{mM})$ in $\mathrm{CDCl}_{3}$ with a water content of $75.49 \mathrm{mM}$ based on ${ }^{1} \mathrm{H} N \mathrm{NR}$ integration. Internal ${ }^{1} \mathrm{H}$ trace (green) is obtained as the vertical sum of the $2 d$ spectrum. External ${ }^{1} H$ and ${ }^{31} P$ traces are plotted from data obtained by separate measurement of the individual nuclear spectra, shown above in Figure S12. 


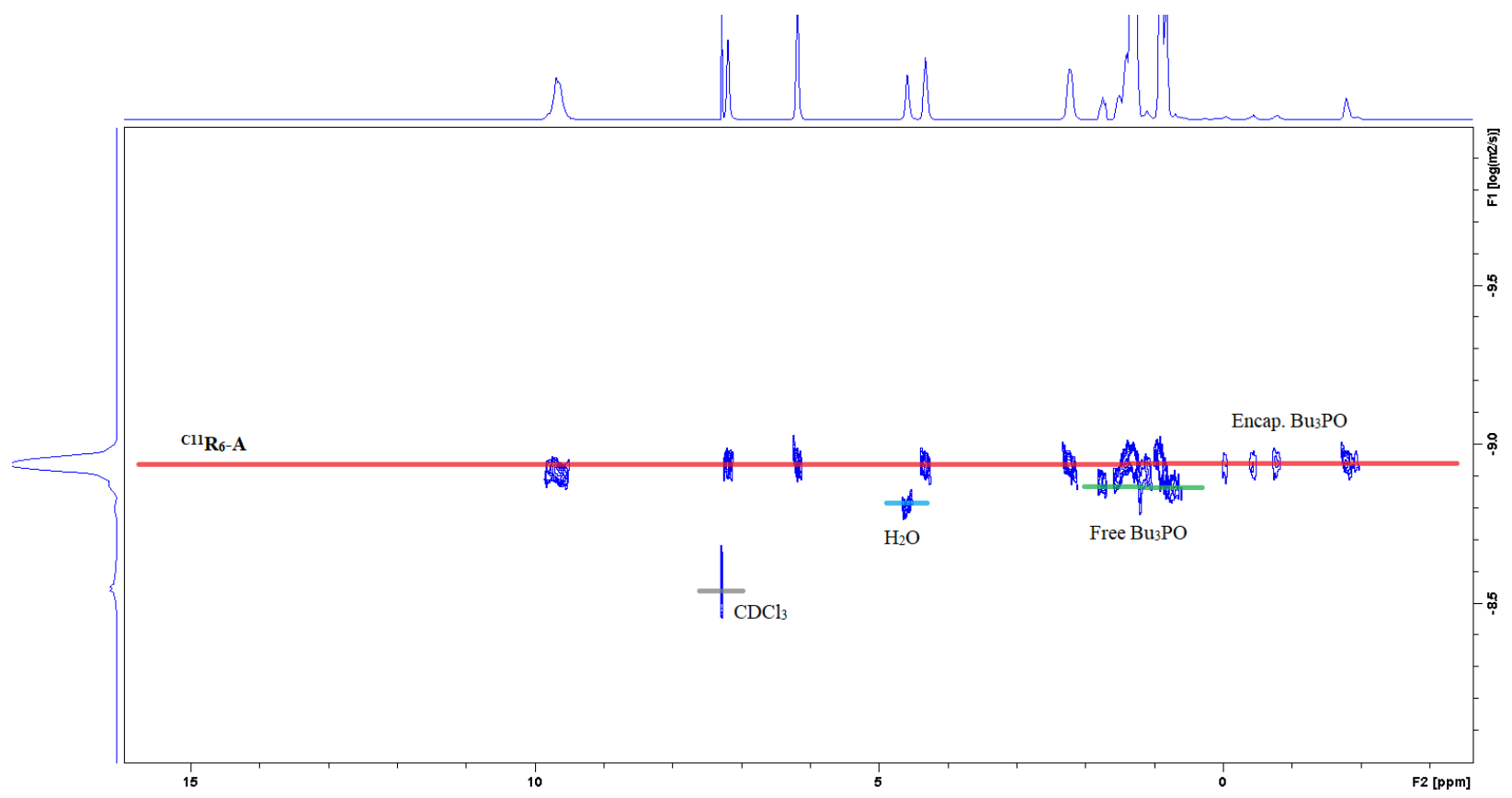

Figure S12. ${ }^{1} \mathrm{H}$ DOSY diffusogram (300 MHz, 19-point decay) of ${ }^{\boldsymbol{C} 11} \boldsymbol{R}_{\mathbf{6}}(5.38 \mathrm{mM})$ and Bus $\mathrm{PO}$ (20 mM) in CDCl with a water content of $75.49 \mathrm{mM}$ determined by ${ }^{1} \mathrm{H} N \mathrm{NR}$. Diffusion was determined by contin fit using Topspin 4.0.9 "DOSY 2d" automation program with a 2-component fitting of the ${ }^{1} \mathrm{H}$ spectra obtained at 19 gradient strengths using a standard LED-type pulse program (ledbpgb2s). Peaks are attributed as annotated, with similar diffusing species highlighted highlighted, ${ }^{C 11} \boldsymbol{R}_{6}-A$ and encapsulated $\mathrm{Bu}_{3} \mathrm{PO}$ (red), free Bu3PO (green), water (light blue), residual $\mathrm{CHCl}_{3}$ (grey). 


\section{Model of water-mediated ${ }^{\mathrm{C} 11} \mathbf{R}_{6}$ formation}

The water dependent equilibrium between ${ }^{\mathbf{C 1 1}} \mathbf{R}_{\mathbf{6}}-\mathbf{A}$ and ${ }^{\mathbf{C} 11} \mathbf{R}_{\mathbf{6}}-\mathbf{B}$ may be modelled by a bimolecular association model for the association of non-structural water. A non-linear fit of the ${ }^{1} \mathrm{H}$ NMR data provides reasonable estimates of the maximum conversion $\left(\theta_{\mathrm{Max}}=0.43 \pm 0.03\right)$ and equilibrium constant $\left(\mathrm{K}_{\mathrm{eq}}=13.17 \pm 1.75 \mathrm{mM}\right)$. For practical purposes the analysis of our data used an empirical function (Equation S1) which includes terms to offset the 8 water molecules for the formation of the capsule.

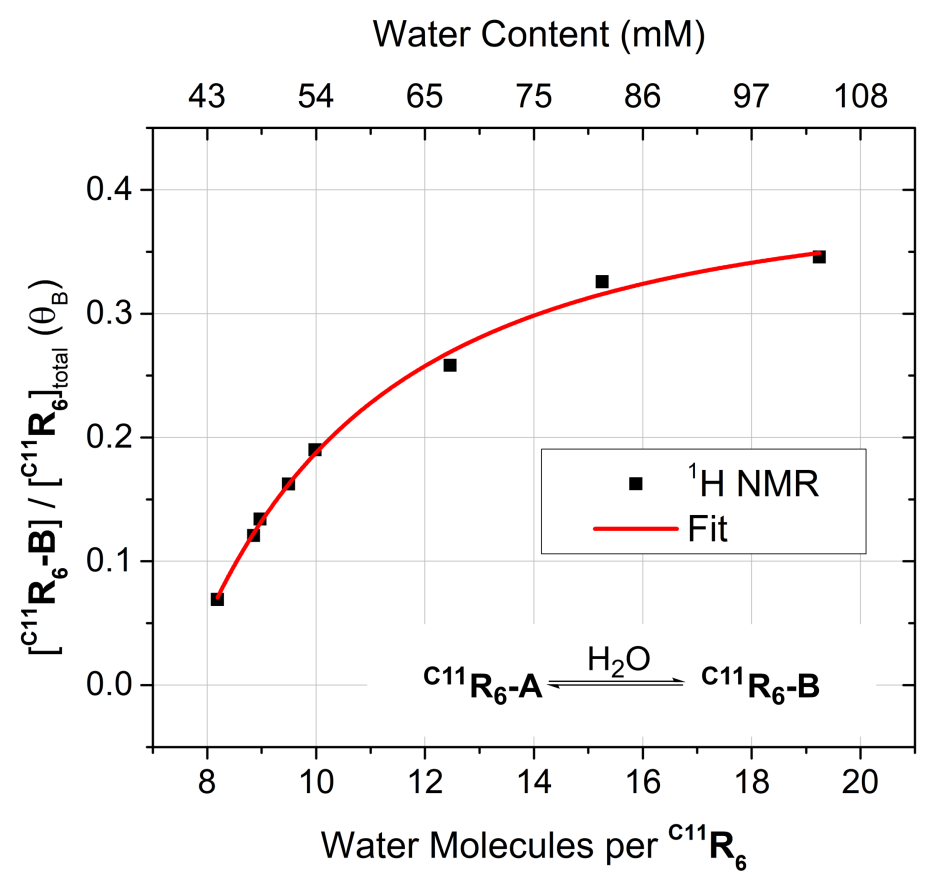

Figure S13. Plot showing the water dependent conversion of ${ }^{C 11} \boldsymbol{R}_{6}-\boldsymbol{A}$ to ${ }^{C 11} \boldsymbol{R}_{6}-\boldsymbol{B}$ with non-linear fit of an empirical model detailed below in Equation S1.

$$
\theta_{\mathrm{B}}=\frac{444.174 * \mathrm{c}_{\mathrm{wat}}}{\mathrm{c}_{\mathrm{wat}}+0.05}-443.578
$$

Equation S1. Empirical function used for modeling of conversion between ${ }^{C 11} \boldsymbol{R}_{6}$-A and ${ }^{C 11} \boldsymbol{R}_{6}-\boldsymbol{B}$ based on the water content $\left(c_{\text {wat }}, m M\right)$ determined by non-linear fitting. 


\section{Models of chemical exchange}

Chemical exchange is often considered either 'fast' when a single peak is observed, or 'slow' when two peaks are apparent, in this section we provide detailed guidance on the NMR observation of chemically exchanging species, in particular the criterion for 'fast' exchange phenomenon, and how they appear in the experimental results of this study.

a.

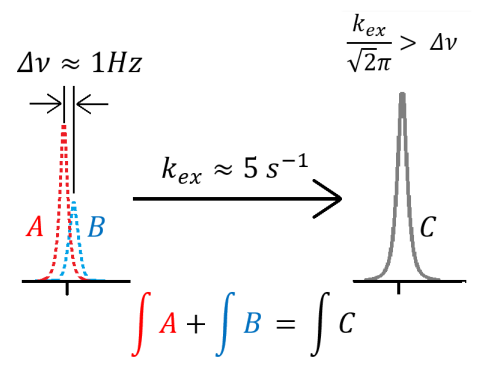

b.

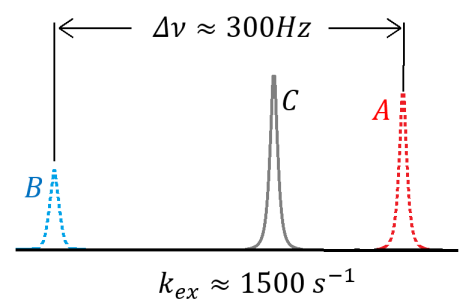

c.

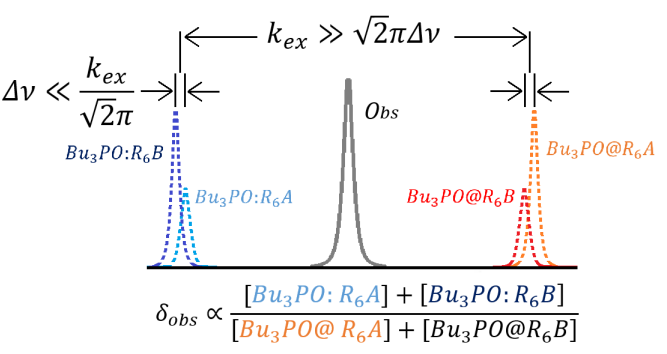

Figure S14. Examples of chemical exchange that appear 'fast' on the NMR time scale. (a) peaks are too similar in frequency to be distinguished, resulting in an observable peak (C). (b) exchange is very rapid, resulting in a single observable peak (C). (c) The combination of the previous two phenomenon as observed in this study.

If chemical exchange between two (or more) states faster than NMR can distinguish between the peaks corresponding to the individual states, then a single peak will be observed in the NMR spectrum that is the superposition of the two or more states - this is referred to as "fast exchange". The limit which NMR may distinguish between peaks is determined by their separation of those peaks (in $\mathrm{Hz}$ ) as provided below in Equation S2.

$$
k_{\text {lim }}=\frac{\Delta v}{\pi \sqrt{2}}
$$

Equation S2. The limit to distinguish peaks for nuclei in chemical exchange by NMR (klim) is a consequence of the difference in peak frequency ( $\Delta v)$, given in $\operatorname{Hertz}\left(\mathrm{Hz}\right.$, or $\left.\mathrm{s}^{-1}\right)$.

This necessitates that if exchanging peaks are too similar in resonance frequency (in $\mathrm{Hz}$ ), then they cannot distinguish even at seemingly slow rates of exchange (Figure S14a), this leads to the appearance of a single peak for aromatic peaks of ${ }^{\mathbf{C 1 1}} \mathbf{R}_{\mathbf{6}}-\mathbf{A}$ and ${ }^{\mathrm{C} 11} \mathbf{R}_{\mathbf{6}}-\mathbf{B}$. Similarly, even if they are well separated, a sufficiently fast exchange will result in a single peak (Figure 14b), as was observed with the association of water. In the case of encapsulated $\mathrm{Bu}_{3} \mathrm{PO}$, the combination of a rapid exchange between hydrogen-bond adducts $\left(\mathrm{Bu}_{3} \mathrm{PO}:{ }^{\mathrm{C} 11} \mathbf{R}_{\mathbf{6}}\right)$ and encapsulated $\left(\mathrm{Bu} 3 \mathbf{P O} @{ }^{\mathrm{C}_{11}} \mathbf{R}_{\mathbf{6}}\right)$ states and the too similar frequencies of the environment within ${ }^{{ }^{111}} \mathbf{R}_{\mathbf{6}}-\mathbf{A}$ or ${ }^{{ }^{11}} \mathbf{R}_{\mathbf{6}}-\mathbf{B}$, results in a single observable peak (Figure S14c) that is the population-weighted superposition of the four states. 


\section{Rearrangement of bridging waters in ${ }^{\mathrm{C} 11} \mathbf{R}_{6}-\mathrm{B}$}

In our CMD simulations we observed rare rearrangement of the incorporated water molecules which form ${ }^{\mathbf{C 1 1}} \mathbf{R}_{\mathbf{6}}$-B, an example of which is shown below in Figure S17. These events were rare with some simulations showing no rearrangements while others featuring several. Because of the rarity of this event, we are unfortunately unable to provide a quantitative analysis of the water bridge rearrangement using CMD.

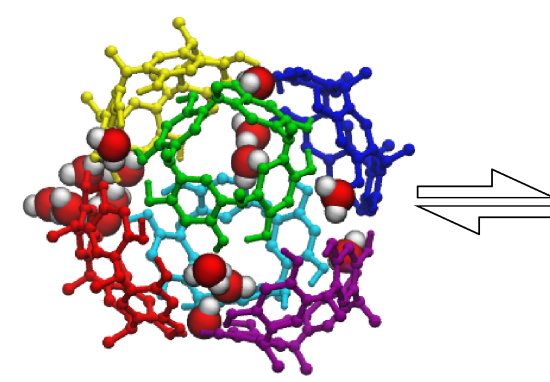

$\mathrm{t}=152 \mathrm{~ns}$

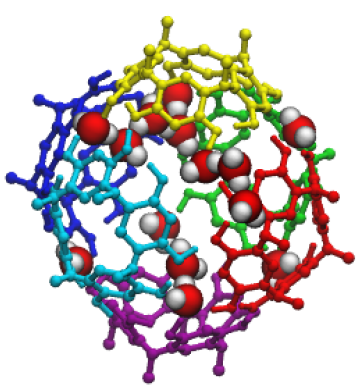

$\mathrm{t}=183 \mathrm{~ns}$
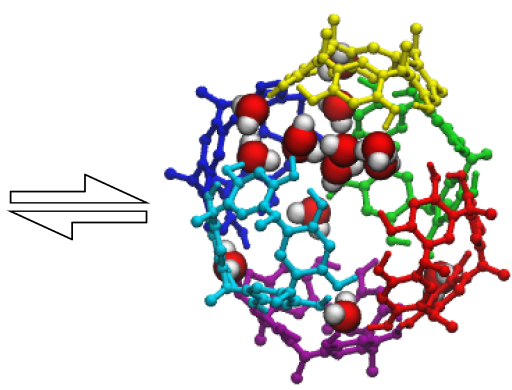

$\mathrm{t}=224 \mathrm{~ns}$

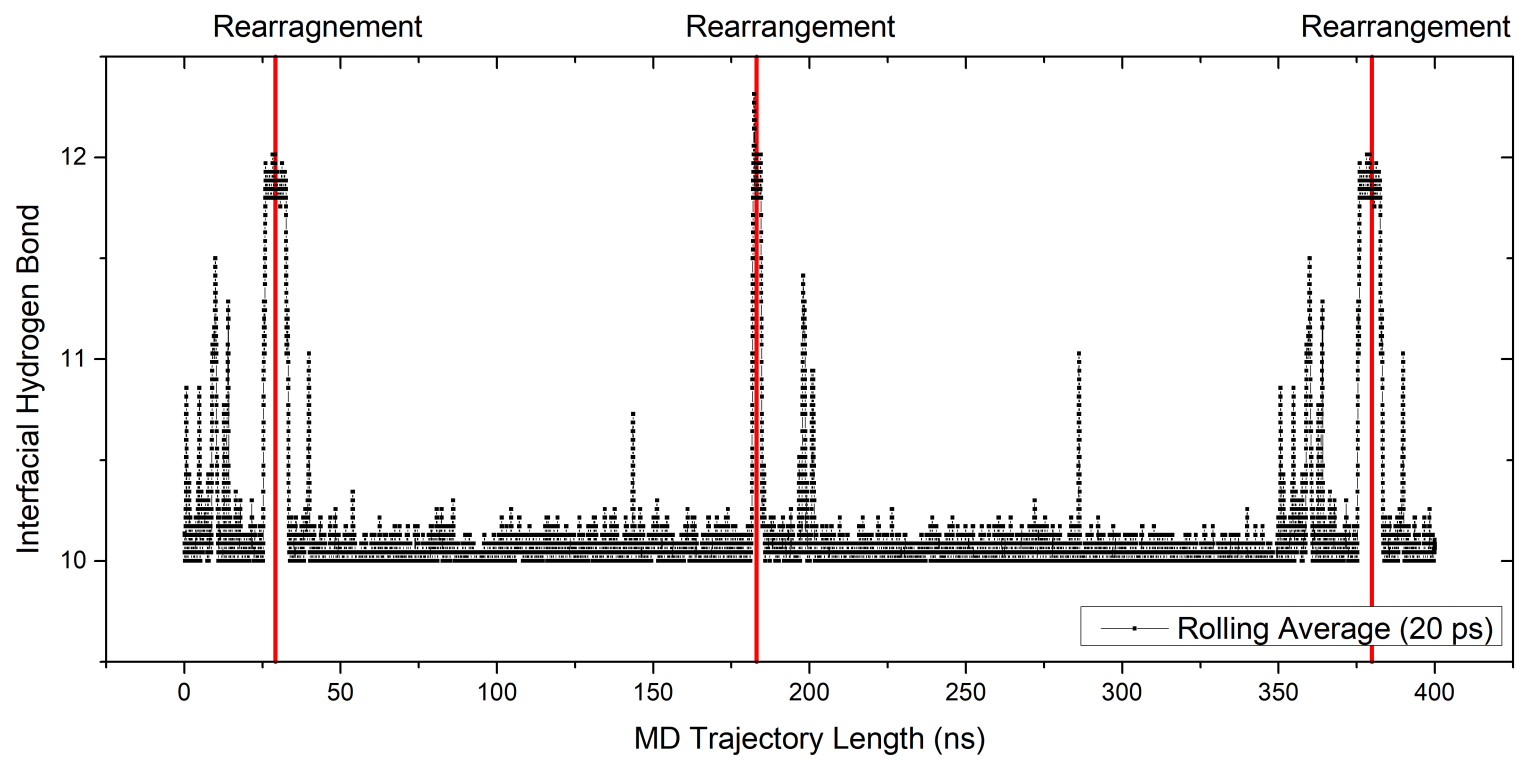

Figure S15. An example of bridging water re-arrangement observed in the ${ }^{C 11} \boldsymbol{R}_{6}$-B form of the capsule by a CMD simulation featuring a total of 18 water molecules. Renderings of individual states before, during, and after the transition of the water bridge (top), alongside a rolling average of the interfacial hydrogen bonds showing three transition events (red lines) over the course of the first $400 \mathrm{~ns}$ of an $800 \mathrm{~ns}$ total CMD trajectory (bottom). The six resorcin[4]arene faces, rendered in a CPK representation, are individually colored to distinguish movement of the incorporated water from the red-yellow edge to the cyan-yellow edge via an intermediate which features encapsulated water molecules (rendered in van der Waal volume spheres colored by atom). These renderings omit alkyl pendant groups and non-hydroxy hydrogen atoms for clarity, though they are included in the CMD models. 


\section{Hydrogen-Bond Network in ${ }^{\mathrm{C} 11} \mathbf{R}_{6}-\mathrm{B}$}

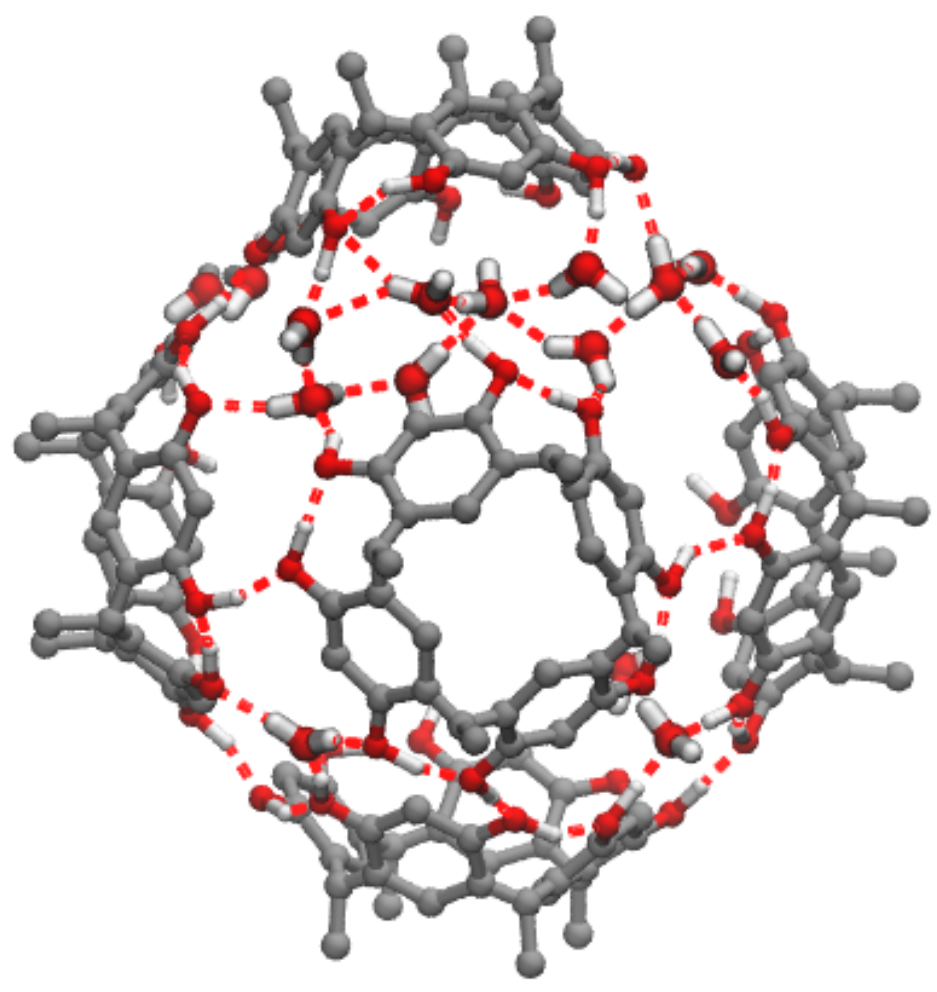

Figure S16. Rendering depicting a typical hydrogen bond network of the associated water molecules found in ${ }^{C 11} \boldsymbol{R}_{6}-\boldsymbol{B}$. Aryl and alkyl hydrogen atoms and alkyl pendant groups, and distant resorcin[4]arene monomer, omitted for clarity. 


\section{Computational Modelling of Sorbyl Alcohol Isomerization.}
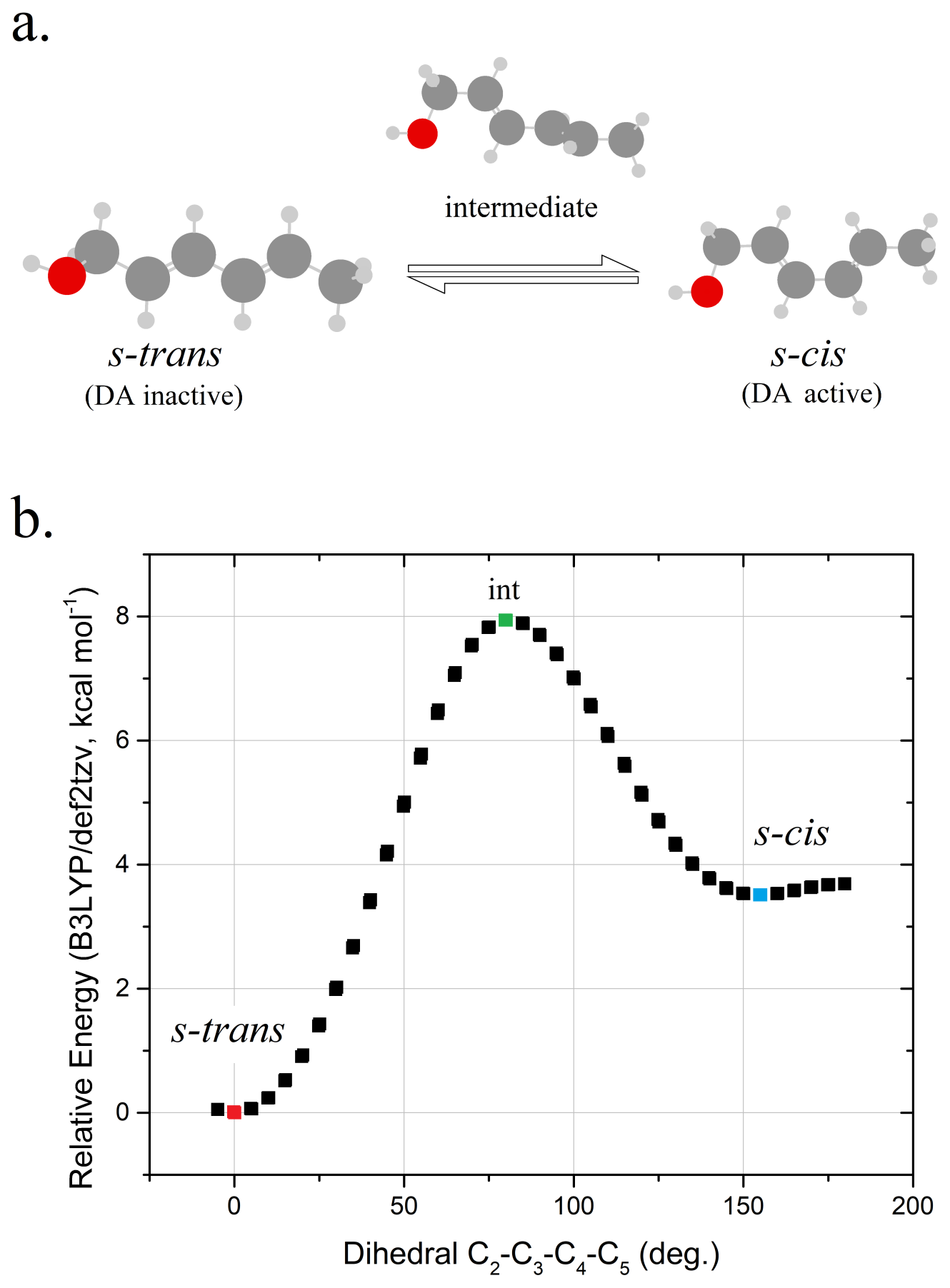

Figure S17. Depiction of the isomerization between Diels-Adler inactive s-trans sorbyl alcohol and its Diels-Adler active s-cis isomer (a), the free energies computed by DFT at a B3LYP/def2tzv theory level obtained from a relaxed energy scan of the central dihedral. Energies for the s-trans (red), intermediate (green) and s-cis isomers are highlighted for reference. The intermediate isomer was optimized separately as a transition state resulting in a minimum barrier of $7.96 \mathrm{kcal} \mathrm{mol}^{-1}$, these calculations revealed a single imaginary vibrational frequency $\left(v_{t s}=-\right.$ $\left.167.50 \mathrm{~cm}^{-1}\right)$, corresponding to the dihedral rotation. 


\section{Model Data for ${ }^{{ }^{11}} R_{1}$ monomer}

Table S2. Mol2 data file with atom information (left) and connectivity (right) for monomer subunit used in MD.

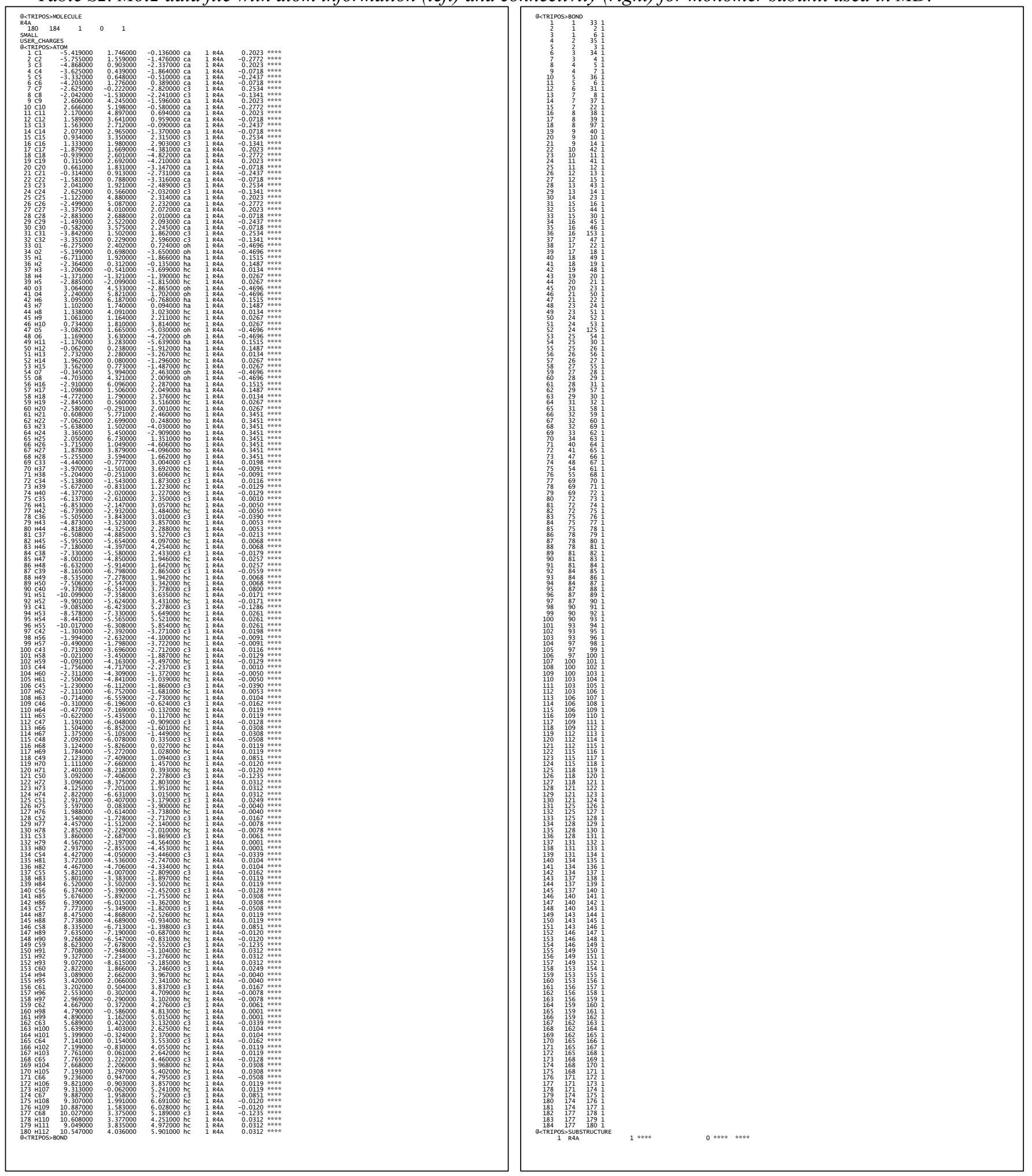


Table S3. Amber forcefield file used during simulations.

\begin{tabular}{|c|c|c|}
\hline \multicolumn{3}{|c|}{ Force field parameters } \\
\hline ca & \multicolumn{2}{|c|}{12.010} \\
\hline c3 & \multirow{2}{*}{\multicolumn{2}{|c|}{12.010}} \\
\hline oh & \\
\hline ha & & \\
\hline hc & \multicolumn{2}{|c|}{$\begin{array}{l}1.008 \\
1.008\end{array}$} \\
\hline ho & \multicolumn{2}{|c|}{$\begin{array}{r}1.008 \\
35.450\end{array}$} \\
\hline c1 & \multirow{2}{*}{\multicolumn{2}{|c|}{$\begin{array}{r}35.450 \\
1.008\end{array}$}} \\
\hline h3 & & \\
\hline $\begin{array}{l}\mathrm{OW} \\
\mathrm{HW}\end{array}$ & \multicolumn{2}{|c|}{$\begin{array}{r}16.000 \\
1.008\end{array}$} \\
\hline \multicolumn{3}{|l|}{ BOND } \\
\hline$c 3-c 3$ & \multirow{11}{*}{$\begin{array}{r}303.100 \\
558.556 \\
247.436 \\
510.522 \\
75.500 \\
337.300 \\
352.587 \\
397.976 \\
0.000 \\
529.580 \\
179.500\end{array}$} & 1.535 \\
\hline ca-ca & & 1.583 \\
\hline $\mathrm{ca}-\mathrm{c} 3$ & & 1.250 \\
\hline ca-oh & & 1.468 \\
\hline c3-c1 & & 1.758 \\
\hline c3-hc & & 1.092 \\
\hline oh-ho & & 1.156 \\
\hline ca-ha & & 0.985 \\
\hline $\mathrm{HW}-\mathrm{HW}$ & & 1.633 \\
\hline $\mathrm{OW}-\mathrm{HW}$ & & 1.012 \\
\hline c3-h3 & & 1.095 \\
\hline \multicolumn{3}{|l|}{ ANGLE } \\
\hline$c 3-c 3-c 3$ & \multicolumn{2}{|c|}{$63.210 \quad 110.630$} \\
\hline ca-ca-oh & $\begin{array}{l}63.210 \\
49.033\end{array}$ & 119.077 \\
\hline $\mathrm{ca}-\mathrm{ca}-\mathrm{c} 3$ & 29.530 & 122.978 \\
\hline ca-ca-ca & \multirow{2}{*}{$\begin{array}{l}47.427 \\
81.176\end{array}$} & 126.032 \\
\hline \multirow{2}{*}{$\begin{array}{l}\mathrm{ca}-\mathrm{c3}-\mathrm{c3} \\
\mathrm{ca}-\mathrm{c3}-\mathrm{ca}\end{array}$} & & 114.295 \\
\hline & & 121.326 \\
\hline$c 7-c 3-c 1$ & 41.400 & 112.200 \\
\hline \multirow{2}{*}{$\begin{array}{l}h c-c 3-h c \\
h c-c 3-c 3\end{array}$} & \multirow{2}{*}{$\begin{array}{l}39.430 \\
46.370\end{array}$} & 108.350 \\
\hline & & 110.050 \\
\hline \multirow{3}{*}{$\begin{array}{l}\text { ca-ca-ha } \\
\text { ca-c3-hc } \\
\text { ca-oh-ho }\end{array}$} & 34.704 & 119.931 \\
\hline & 78.729 & 111.016 \\
\hline & 24.376 & 107.232 \\
\hline $\begin{array}{l}\text { Ca-oh-ho } \\
\text { HW-OW-HW }\end{array}$ & 37.950 & 113.240 \\
\hline$c 7-c 3-h 3$ & 28.700 & 107.660 \\
\hline
\end{tabular}

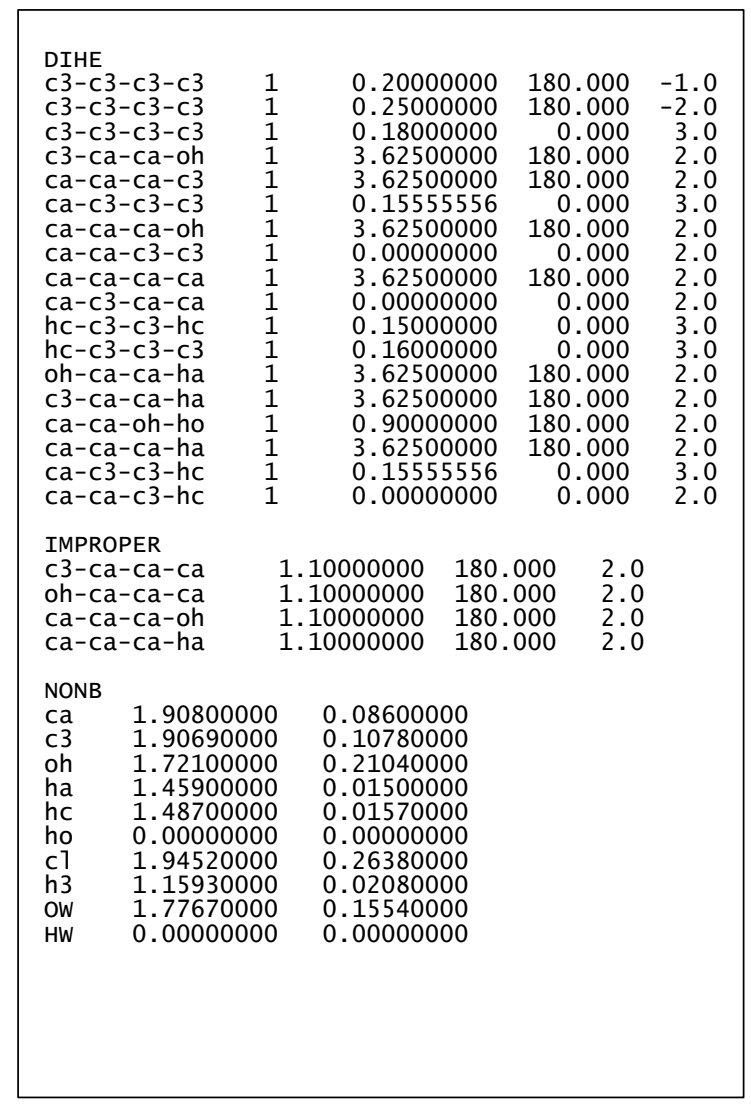

\section{Supplementary Material.}

monomer.mol2 - Contains charges, coordinates and connectivity of monomer (Table S2)

sim.frcmod - Contains forcefield parameters in Amber format (Table S3) 


\section{References}

1. Frisch, M. J.; Trucks, G. W.; Schlegel, H. B.; Scuseria, G. E.; Robb, M. A.; Cheeseman, J. R.; Scalmani, G.; Barone, V.; Petersson, G. A.; Nakatsuji, H.; Li, X.; Caricato, M.; Marenich, A. V.; Bloino, J.; Janesko, B. G.; Gomperts, R.; Mennucci, B.; Hratchian, H. P.; Ortiz, J. V.; Izmaylov, A. F.; Sonnenberg, J. L.; Williams-Young, D.; Ding, F.; Lipparini, F.; Egidi, F.; Goings, J.; Peng, B.; Petrone, A.; Henderson, T.; Ranasinghe, D.; Zakrzewski, V. G.; Gao, J.; Rega, N.; Zheng, G.; Liang, W.; Hada, M.; Ehara, M.; Toyota, K.; Fukuda, R.; Hasegawa, J.; Ishida, M.; Nakajima, T.; Honda, Y.; Kitao, O.; Nakai, H.; Vreven, T.; Throssell, K.; Montgomery, J. A., Jr.; Peralta, J. E.; Ogliaro, F.; Bearpark, M. J.; Heyd, J. J.; Brothers, E. N.; Kudin, K. N.; Staroverov, V. N.; Keith, T. A.; Kobayashi, R.; Normand, J.; Raghavachari, K.; Rendell, A. P.; Burant, J. C.; Iyengar, S. S.; Tomasi, J.; Cossi, M.; Millam, J. M.; Klene, M.; Adamo, C.; Cammi, R.; Ochterski, J. W.; Martin, R. L.; Morokuma, K.; Farkas, O.; Foresman, J. B.; Fox, D. J. Gaussian 16 Revision C.01, Gaussian Inc., Wallingford CT, 2016.

2. Wang, J.; Wang, W.; Kollman P. A.; Case, D. A. Automatic atom type and bond type perception in molecular mechanical calculations. J. Mol. Graph. Model. 2006, 25, 247260.

3. Bannwarth, C.; Ehlert, S.; Grimme, S. GFN2-xTB-An Accurate and Broadly Parametrized Self-Consistent TightBinding Quantum Chemical Method with Multipole Electrostatics and Density-Dependent Dispersion Contributions. J. Chem. Theory Comput. 2019, 15 (3), 1652-1671, DOI: 10.1021/acs.jctc.8b01176.

4. Fox, T., \& Kollman, P. A. Application of the RESP Methodology in the Parametrization of Organic Solvents. J. Phys. Chem. B 1998, 102(41), 8070-8079, DOI:10.1021/jp9717655.

5. Betz, R. M., \& Walker, R. C. Paramfit: Automated optimization of force field parameters for molecular dynamics simulations. J. Comput. Chem. 2014, 36(2), 79-87, DOI:10.1002/jcc.23775.

6. Wang, J., Wolf, R. M.; Caldwell, J. W.;Kollman, P. A.; Case, D. A. Development and testing of a general AMBER force field. J. Comput. Chem. 2004, 25, 1157-1174.

7. Rappe, A. K.; Casewit, C. J.; Colwell, K. S.; Goddard, W. A. III; Skiff, W. M. UFF, a full periodic table force field for molecular mechanics and molecular dynamics simulations. J. Am. Chem. Soc. 1992, 114, 10024-10039.

8. Martínez, L.; Birgin, R.; Birgin, E. G.; Martínez, J. M. Packmol: A package for building initial configurations for molecular dynamics simulations. J. Comput. Chem. 2009, 30(13), 2157-2164.

9. Wu, Y.; Tepper, H. L.; Voth, G. A. Flexible simple point-charge water model with improved liquid-state properties. J. Chem. Phys. 2006, 124(2), 024503, DOI:10.1063/1.2136877

10. Case, D. A.; Cerutti, D. S.; Cheatham, T. E., III; Darden, T. A.; Duke, R. E.; Giese, T. J.; Gohlke, H.; Goetz, A. W.; Izadi, S.; Janowski, P.; Kaus, J.; Kovalenko, A.; Lee, T. S.; LeGrand, S.; Li, C.; Lin, T. L., Luo, R.; Madej, B.; Mermelstein, D.; Merz, K. M.; Monard, G.; Nguyen, H.; Nguyen, I.; Omelyan, A. O., Roe, D. R.; Roitberg, A.; Sagui, C.; Simmerling, C. L.; Botello-Smith, W. M.; Swails, J.; Walker, J. W., Wolf, R. M.; Wu, X.; Xiao, L.; Kollman, P. A. AMBER 2016, University of California, San Francisco, 2016.

11. Salomon-Ferrer, R.; Goetz, A.W.; Poole, D.; Le Grand, S.; Walker, R.C. Routine microsecond molecular dynamics simulations with AMBER on GPUs. 2. Explicit solvent Particle Mesh Ewald. J. Chem. Theory Comput. 2013 9, 38783888 .

12. Ramsey, S.; Nguyen, C.; Salomon-Ferrer, R.; Walker, R. C.; Gilson, M. K.; Kurtzman, T. Solvation thermodynamic map-ping of molecular surfaces in AmberTools: GIST. J. Comput. Chem. 2016, 37 (21), 2029-2037, DOI: $10.1002 /$ jcc.24417.

13. Elidrisi,I. ; Negin, S.; Bhatt, P. V.; Govender, T.; Kruger, H. G.; Gokel, G. W.; Maguire, G. E. M. Pore formation in phospholipid bilayers by amphiphilic cavitands. Org. Biomol. Chem. 2011, 9, 4498-4506, DOI: 10.1039/C0OB01236J.

14. Stirling, C. J. M.; Fundin, L. J.; Williams, N. H. Trimorphism in solid resorcinarenes. Chem. Commun. 2007, (17), 1748-1750, DOI: 10.1039/b700469a.

15. Payne, R. M.; Oliver, C. L. A propanol-seamed C-methylcalix[4]resorcinarene hexamer accessible via solution crystallization, liquid-assisted grinding and vapour sorption. CrystEngComm 2018, 20 (14), 1919-1922, DOI:10.1039/c8ce00294k. 